\title{
Sampling Strategies Near a Low-Order Detonation and a Target at an Artillery Impact Area
}

Thomas F. Jenkins, Alan D. Hewitt, Thomas A. Ranney,

November 2004

Charles A. Ramsey, Dennis J. Lambert, Kevin L. Bjella,

and Nancy M. Perron 
ERDC/CRREL TR-04-14

November 2004

\section{Sampling Strategies Near a Low-Order Detonation and a Target at an Artillery Impact Area}

Thomas F. Jenkins, Alan D. Hewitt, Thomas A. Ranney, Charles A. Ramsey, Dennis J. Lambert, Kevin L. Bjella, and Nancy M. Perron

Approved for public release; distribution is unlimited. 


\section{ABSTRACT}

Field sampling experiments were conducted at the firing range at Fort Polk, Louisiana. The objectives were to determine the spatial distribution and best approach for collecting representative surface soil samples to estimate mean concentrations of residues of high explosives at two types of potential source zones: (1) an area near a low-order [partial] detonation of an 81-mm mortar and (2) an artillery/mortar target. Soil sampling near the low-order detonation revealed the presence of potential "hot spots" and showed that the concentrations of RDX and TNT ranged over five orders of magnitude. The range of concentrations was reduced to a factor of about 60 when randomly collected 25 -increment composite samples were collected within this area. The range reduced further to about a factor of three for four simulated (i.e., existing discrete values) 25 -increment systematically derived composite samples. Thus a vast improvement in the repeatability of replicate samples can be achieved using composite sampling approaches. Composite samples collected around a target showed that the distribution of energetic residues was random and overall the concentrations were much lower than around the partially detonated round.

DISCLAIMER: The contents of this report are not to be used for advertising, publication, or promotional purposes. Citation of trade names does not constitute an official endorsement or approval of the use of such commercial products. All product names and trademarks cited are the property of their respective owners. The findings of this report are not to be construed as an official Department of the Army position unless so designated by other authorized documents. 


\section{CONTENTS}

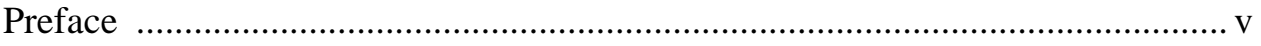

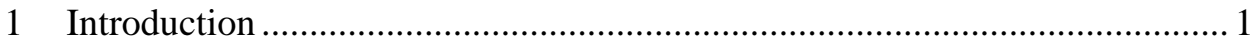

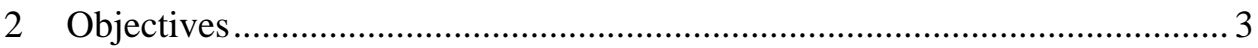

3 Soil Sample Collection and Analysis ........................................................... 4

Soil sample collection in an area near a low-order detonation of an

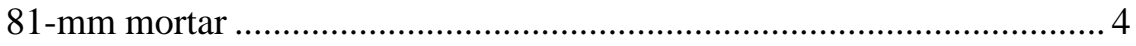

Sampling near a tank target uphill from the $10-\mathrm{m} \times 10$-m grid...................... 6

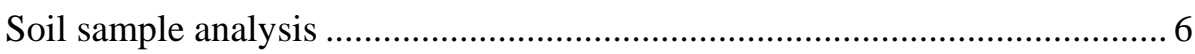

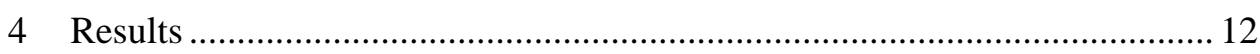

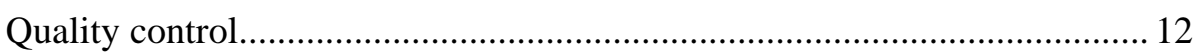

Grid samples from the area near a low-order 81-mm mortar detonation ...... 14

Comparison of field duplicate discrete and ten-increment composites for

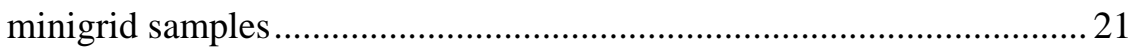

Results for 25-increment composite samples collected within the 10-m $\times 10$-m grid near low-order detonation................................................ 24

Line composite samples surrounding $10-\mathrm{m} \times 10$-m grid.............................. 28

Physical size of hot spot from low-order 81-mm mortar round ................... 28

Target analyte concentrations near an artillery target ................................... 31

5 Summary and Conclusions ........................................................................... 33

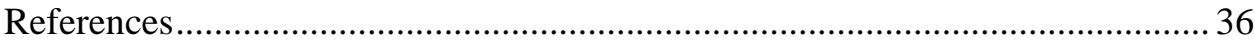

\section{ILLUSTRATIONS}

Figure 1. Chunks of Composition B from the partial detonation of an 81-mm mortar round found in the artillery impact area at Fort Polk, Louisiana ..... 4

Figure 2. Sampling a $10-\mathrm{m} \times 10$-m grid area encompassing pieces of chunk explosive from a low-order 81-mm mortar round. The tank target is uphill and in the background

Figure 3. Relationship of a $10-\mathrm{m} \times 10$-m sampling grid and a tank target in the artillery impact area at Fort Polk, Louisiana ....

Figure 4. $10-\mathrm{m} \times 10$-m sampling grid subdivided into $100-\mathrm{m}^{2}$ minigrids and linear sampling lines around the major grid area 
Figure 5. Coring device used to collect soil samples at Fort Polk, Louisiana ....... 9

Figure 6. Collecting and weighing visible pieces of Composition B found around a partial detonation of an 81-mm mortar ................................................... 9

Figure 7. Distribution of soil RDX concentrations from 100 discrete soil samples taken in the 1 - $\mathrm{m} \times 1$-m minigrids

Figure 8. Distribution of the log soil RDX concentrations from 100 discrete soil samples taken from 1-m × 1-m minigrids ................................................. 19

Figure 9. Soil concentration of RDX relative to its location within the $10-\mathrm{m} \times 10$ $m$ grid

Figure 10. Weight of Composition B and soil RDX concentration and their relative position in the sampling grid

Figure 11. Soil RDX concentrations in linear composite samples taken at various distances from the sampling grid.

Figure 12. Soil concentration of RDX taken in various sampling areas around a

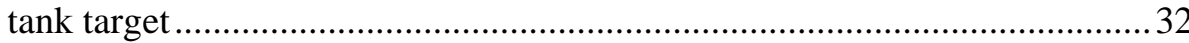

\section{TABLES}

Table 1. Explosives detection limits for soil and water .................................... 11

Table 2. Quality control soil samples for Fort Polk study ................................... 12

Table 3. Analytical results for Fort Polk samples that were analyzed, reground, and subsamples analyzed in triplicate....

Table 4. Results from the analysis of discrete samples from 1001 -m $\times 1-m$ minigrids in an area near a low-order 81-mm mortar detonation at the impact range at Fort Polk

Table 5. Results from the analysis of duplicate discrete and ten-increment composite samples from 20 randomly chosen minigrids near location of low-order mortar detonation

Table 6. Results from the analysis of 25-increment composite samples from grid near a low-order 81-mm mortar detonation at Fort Polk

Table 7. Comparison of concentration estimates for target analytes using various collection strategies in a $10-\mathrm{m} \times 10$-m grid near a low-order detonation...... 26

Table 8. Calculation of total mass of RDX in 10-m $\times 10$-m sampling grid at Fort Polk, Louisiana, and its potential for groundwater contamination ........27

Table 9. Concentrations of explosives residues in ten-increment line composite samples collected from the four edges of $10-\mathrm{m} \times 10$-m grid.........................29

Table 10. Target analyte concentrations in area around an artillery target in the impact area, Fort Polk ........................................................................ 31 


\section{PREFACE}

This report was prepared by Dr. Thomas F. Jenkins, Alan D. Hewitt, and Kevin L. Bjella, Environmental Sciences Branch, U.S. Army Engineer Research and Development Center (ERDC), Cold Regions Research and Engineering Laboratory (CRREL), Hanover, New Hampshire; Thomas A. Ranney, Science and Technology Corporation, Hanover, New Hampshire; Charles A. Ramsey, EnviroStat, Inc., Fort Collins, Colorado; Dennis J. Lambert, Engineering Resources Branch, ERDC-CRREL; and Nancy M. Perron, Snow and Ice Branch, ERDC-CRREL.

Funding for this work was provided under Project CP1155 by the Strategic Environmental Research and Development Program (SERDP), Mr. Bradley Smith, Executive Director, Dr. Jeffrey Marqusee, Technical Director, and Dr. Robert Holst, Project Monitor. Dr. Judith C. Pennington, U.S. Army ERDC, Environmental Laboratory (EL), Vicksburg, Mississippi, was the Principal Investigator for Project CP1155.

The authors acknowledge John Buck, U.S. Army Environmental Center (AEC), Aberdeen Proving Ground (APG), Maryland, and Barrett Borrey, U.S. Army Center for Health Protection and Preventive Medicine, (CHPPM), APG, Maryland, and Dr. Charles Stagg, the Installation Environmental Director, for allowing the authors to participate in the Fort Polk Regional Range Study. These organizations provided logistics and EOD support and developed the safety plan that allowed the authors to conduct their research. This study could not have been conducted without their support and collaboration. The authors also express their appreciation for the support given by Greg Prudhomme and Dennis Jaffery of the Environmental and Natural Resources Management Division at Fort Polk.

The authors also thank Dr. Jeffrey Davis, ERDC-EL, for assistance in sampling at Fort Polk, Louisiana, and thank Pete Garcia, Russ Chattles, Chuck Brewer, and Lee Wallace from EOTI Corporation for assistance in sampling and for providing EOD support during the site sampling activities.

This report was technically reviewed by Marianne E. Walsh, CRREL, and Dr. Clarence L. Grant, Professor Emeritus, University of New Hampshire.

The Commander of the Engineer Research and Development Center is Colonel James R. Rowan, EN. The Director is Dr. James R. Houston. 


\title{
Sampling Strategies Near a Low-Order Detonation and a Target at an Artillery Impact Area
}

\author{
THOMAS F. JENKINS, ALAN D. HEWITT, THOMAS A. RANNEY, \\ CHARLES A. RAMSEY, DENNIS J. LAMBERT, \\ KEVIN L. BJELLA, AND NANCY M. PERRON
}

\section{INTRODUCTION}

A series of papers has been published describing the difficulty in collecting soil samples representative of the mean analyte concentrations at areas contaminated with residues of energetic compounds (Ampleman et al. 2003a, b; Jenkins et al. 1996, 1997, 1999, 2001, 2004; Pennington et al. 2001, 2002, 2003; Thiboutot et al. 1998, 2003; Walsh et al. 2001, 2004). This difficulty is because residues often exist as particulates and are distributed heterogeneously on the surface. Because such particulate residues may serve as the major source of potential off-site migration of these compounds, it can be important to estimate the mass of energetic materials present in these surface soils.

There are a number of different types of DoD training ranges where various types of munitions are used. These include artillery and mortar range impact areas, bombing ranges, antitank rocket range impact areas, demolition ranges, 40$\mathrm{mm}$ rifle grenade ranges, hand grenade ranges, and firing points for the various weapons. The chemical and physical characteristics of energetic residues at these ranges differ substantially. For example, at antitank rocket ranges, nitroglycerin (NG) is deposited near the firing point and is present at the highest concentrations behind the firing line due to back blast. The energetic residue present at the highest concentrations in the impact area of this type of range is mainly 1,3,5,7octahydro-1,3,5,7-tetranitrotetrazocine (HMX) from physically breached casings of the antitank rocket's warhead (Jenkins et al. 1999). For artillery range impact areas, however, the major residues are either 2,4,6-trinitrotoluene (TNT) or Composition B (60\% 1,3,5-hexahydro-1,3,5-trinitrotriazine [RDX], 39 \% TNT) from the warheads of artillery or mortar rounds. The major residues at artillery ranges reside as distributed sources associated with rounds that have undergone low-order (partial) detonation (Pennington et al. 2002, 2003). 
A study was conducted at Canadian Force Base-Valcartier (CFB-Valcartier) in which various sampling protocols were evaluated with regard to their ability to provide representative samples that would provide estimates of mean concentration (Jenkins et al. 2004). Both discrete and multi-increment composite samples were collected within 10 -m $\times 10$-m areas at the firing point and impact area where training is conducted with antitank rockets. The largest sampling problem to be overcome was that residues were distributed heterogeneously over the area, resulting in a range of concentrations of greater than two orders of magnitude in discrete samples collected at both the firing point (NG) and impact area (HMX). From these results it is clear that collection of discrete samples to estimate a mean concentration for areas as small as 100 square meters is very unreliable and the individual values will underestimate the mass of residue loading in most cases. 


\section{OBJECTIVES}

The major objective of this work was to thoroughly characterize an area within an artillery range where the major contamination source was a low-order detonation of a mortar or artillery round to understand the distribution of residues of high explosives. Specifically an attempt was made to estimate the short-range heterogeneity in analyte concentrations and investigate various alternatives to obtain samples representative of the mean analyte concentrations within this type of area. Previous work has shown that low-orders are an anomaly even in highuse target areas. Therefore, in general, the concentrations of energetic residues are anticipated to be very low (Jenkins et al. 1998, 2001; Pennington et al. 2001, 2002, 2003). However, when rounds low-order, a hot spot of residue concentrations is often created, thereby presenting a unique sampling challenge.

A second objective was to collect surface soil samples spatially around an artillery target to determine whether residue concentrations were distributed in a similar manner as found for targets at antitank ranges. For antitank ranges, sampling studies at a number of installations have shown that there is a strong concentration gradient for HMX with the highest concentrations next to the target (Jenkins et al. 1997, 1998; Pennington et al. 2002; Thiboutot et al. in press). 


\section{SOIL SAMPLE COLLECTION AND ANALYSIS}

\section{Soil sample collection in an area near a low-order detonation of an 81-mm mortar}

This study was conducted at an artillery impact range at Fort Polk, Louisiana, from 25 June to 29 June 2003. Initially all field personnel accompanied the AEC/ CHPPM sampling team as they traversed the impact range on foot to collect soil samples at a number of predetermined locations according to their stratified random sampling strategy. At an access road that was downhill from an artillery target, small chunks of what appeared to be explosives residue were observed on the soil surface (Fig. 1). Using the EXPRAY kit (a field test kit designed to detect and classify energetic residues [Plexus Scientific, Silver Spring, Maryland]), these chunks were shown to contain both a nitroaromatic (probably TNT) and a nitramine/nitrate ester (probably RDX) in the chunks of residue found on the ground. Subsequent laboratory analysis at CRREL confirmed the proper ratio of RDX/TNT for Composition B. After inspecting the surface of the soil in the vicinity of these chunks, additional pieces of explosives material were observed. The presence of a fin and casing fragment indicated that the explosives residues originated from an 81-mm mortar that had undergone a low-order detonation.

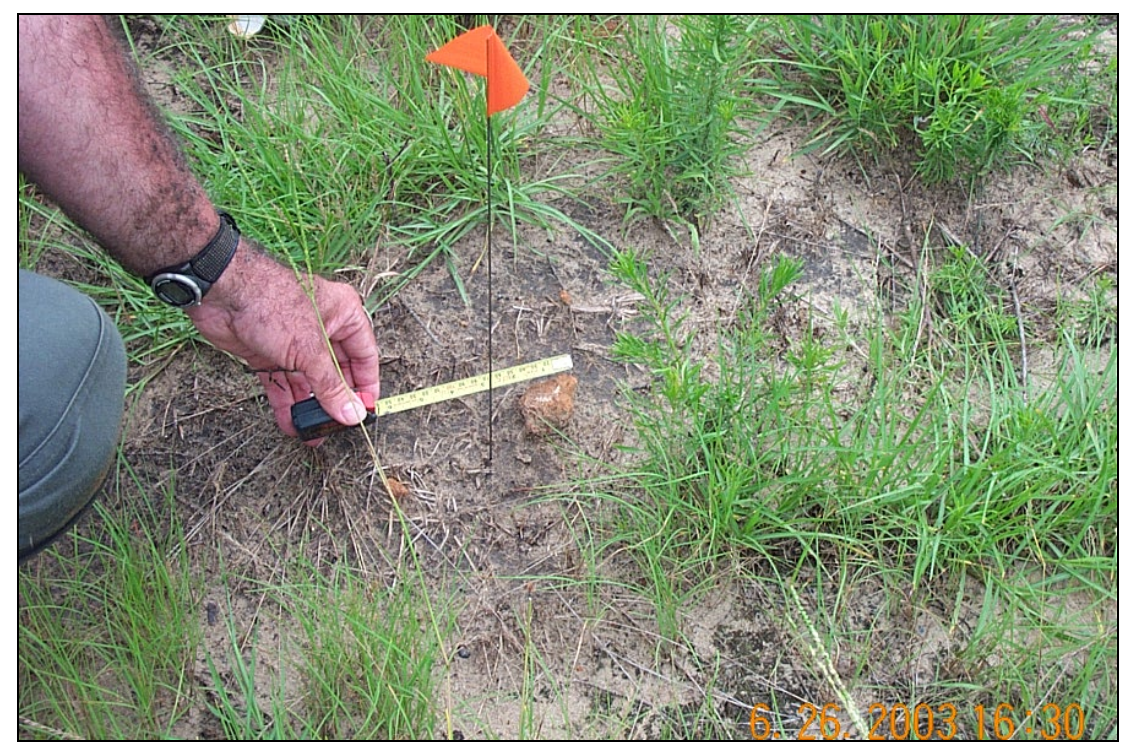

Figure 1. Chunks of Composition B from the partial detonation of an 81-mm mortar round found in the artillery impact area at Fort Polk, Louisiana. 


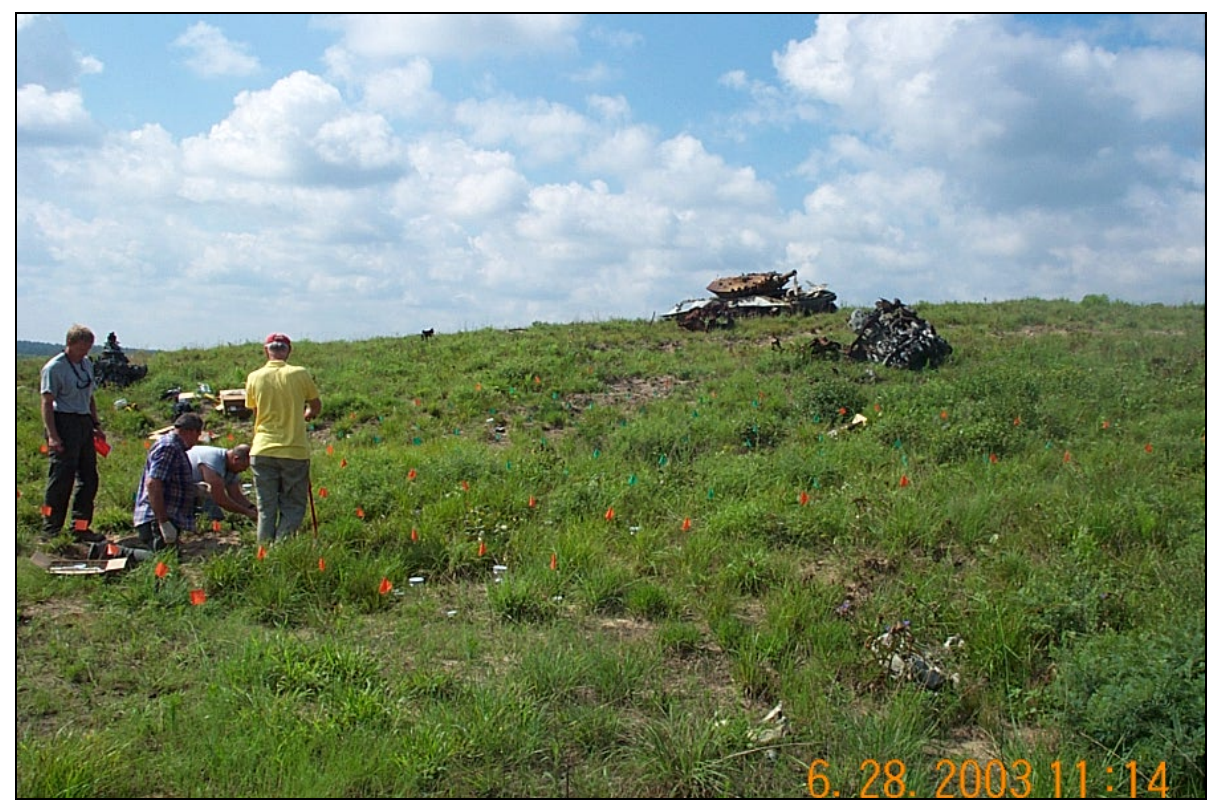

Figure 2. Sampling a 10-m $\times 10-\mathrm{m}$ grid area encompassing pieces of chunk explosive from a low-order 81-mm mortar round. The tank target is uphill and in the background.

Because we were interested in studying the distribution of explosives residues near low-order detonations, we located a $10-\mathrm{m} \times 10$-m sampling grid along the road, encompassing the pieces of chunk explosive that we had visually identified (Fig. 2). The center of this grid was about $30 \mathrm{~m}$ downslope from a heavily used tank target. A diagram of the area is shown in Figure 3. From the surface topography we do not believe that any surface runoff from the tank target located upslope and left of this grid (as seen from the road) would pass over this grid.

The 10-m $\times 10$-m sampling grid was further subdivided into $1001-\mathrm{m} \times 1$-m minigrids (Fig. 4). Within each minigrid a discrete sample was collected from the surface and stored in a 4-oz amber glass jar. These samples and all subsequent samples were obtained using a coring device (Fig. 5) developed at CRREL (Walsh 2004). All core sample increments were of the top $2.5 \mathrm{~cm}$ and were 4.8 $\mathrm{cm}$ in diameter. If visible pieces of explosive were present in a given minigrid, the material was gathered and weighed with a portable balance (Fig. 6), and we collected the soil sample adjacent to where the largest piece of solid explosive had been located. In minigrids that did not have visible solid explosive, samples were collected at a randomly selected location within the $1-\mathrm{m} \times 1-\mathrm{m}$ area. Duplicate discrete soil samples were collected adjacent to the initial discrete sampling location in 20 randomly selected minigrids. Within these same 20 randomly selected minigrids, a ten-increment composite was also collected at 
randomly selected locations and stored in a 32-oz glass jar. The piece of the loworder 81-mm mortar (tail fin and casing fragment) that was observed was located at the boundary of minigrids 3 and 13 .

After all of the visible explosive was removed, and the minigrid discrete and ten-increment composite samples were collected, ten 25-increment composite samples were collected from the entire $10-\mathrm{m} \times 10$-m grid using a random sampling strategy (moving in random directions after the collection of each increment, i.e., analogous to random number generator). Five different individuals collected these samples using the soil corers described above. Samples were stored in 64-oz glass jars.

Beyond the boundaries of the 10 -m $\times 10$-m grid, ten-increment composite samples were collected from lines parallel to and outward from the four sides of the grid at distances of $2 \mathrm{~m}, 5 \mathrm{~m}$, and $10 \mathrm{~m}$ as shown in Figure 4. These composite samples were stored in 32-oz glass bottles.

\section{Sampling near a tank target uphill from the 10-m $\times 10-\mathrm{m}$ grid}

A heavily impacted artillery target was located about $30 \mathrm{~m}$ uphill and to the left (as seen from the road) of the $10-\mathrm{m} \times 10$-m sampling grid. Twelve sampling grids were established as shown in Figure 3 . The boundaries of the four inside grids were from the perimeter of the tank target to $2 \mathrm{~m}$ from the edge. The eight outer grids were located from $2 \mathrm{~m}$ to $5 \mathrm{~m}$ from the edge of the target. A set of ten-increment surface soil composite samples was collected from within grid boundaries using a random sampling strategy similar to that described above. These composite samples were stored in 32-oz glass bottles.

\section{Soil sample analysis}

Soil samples were returned to CRREL and air-dried at room temperature. The discrete and composite samples were processed differently because the sample masses were different.

Discrete samples were dried in 4-oz amber containers, weighed, passed through a \#10 (2-mm) sieve to remove oversize material, the sieved portion weighed, and returned to the 4-oz containers. Discrete samples were not subsampled, rather the entire sample was extracted as follows. A volume of acetonitrile in milliliters (mL), approximately double the mass of the sample in grams, was added to each 4-oz jar unless the sample was too large (greater than $60 \mathrm{~g}$ ) (Hewitt and Walsh 2003). For those cases the sample was transferred to an 8-oz jar and acetonitrile was added. All jars were capped and placed on a tabletop shaker overnight (18 hours @ 150 rpm). The samples were removed from the 
shaker and allowed to settle for at least an hour. An aliquot of each extract was filtered through a $0.45-\mu \mathrm{m}$ Millex FH filter and placed in a 7-mL amber glass vial. Vials were stored in a refrigerator until analyzed.

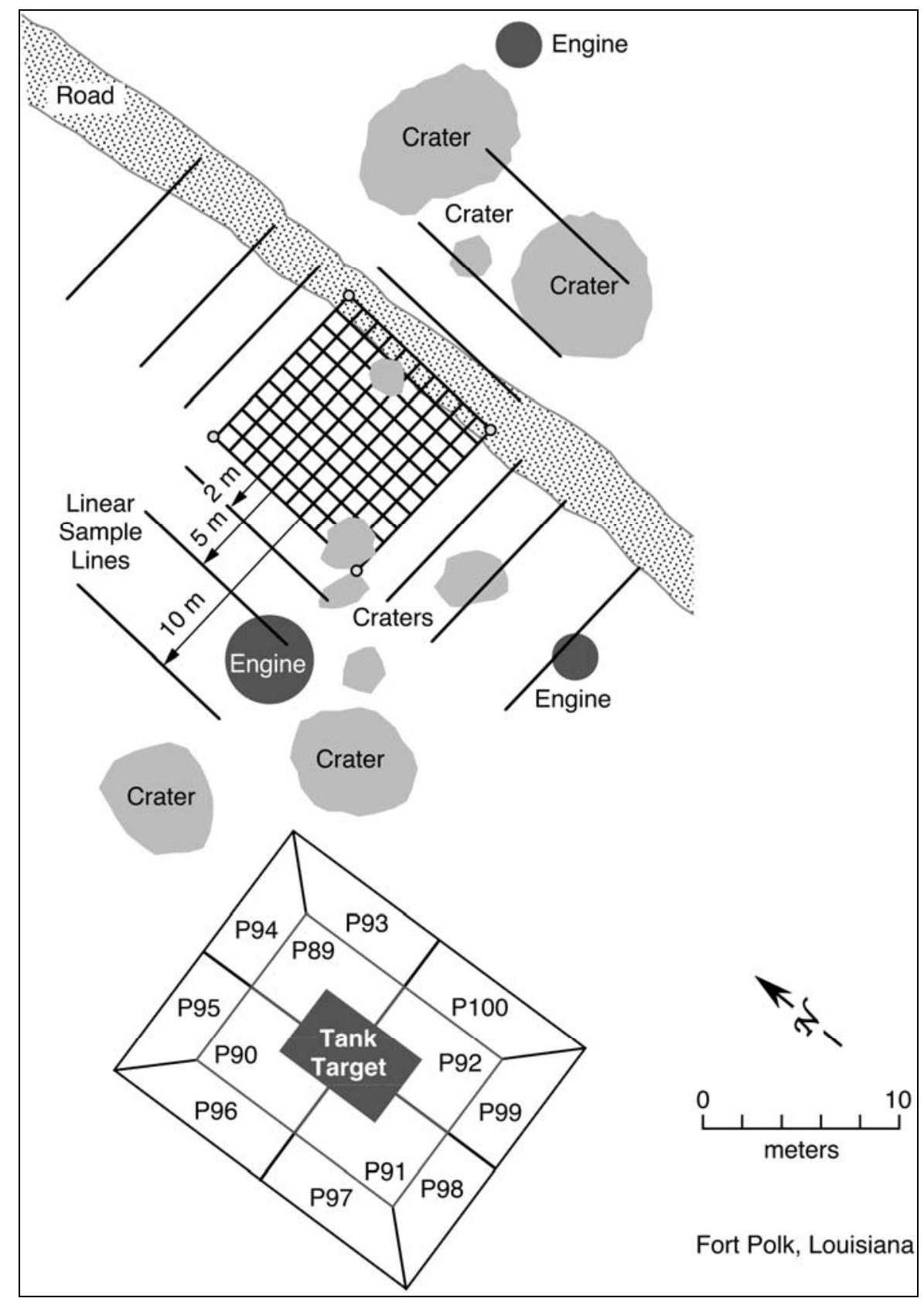

Figure 3. Relationship of a $10-\mathrm{m} \times 10-\mathrm{m}$ sampling grid and a tank target in the artillery impact area at Fort Polk, Louisiana. 


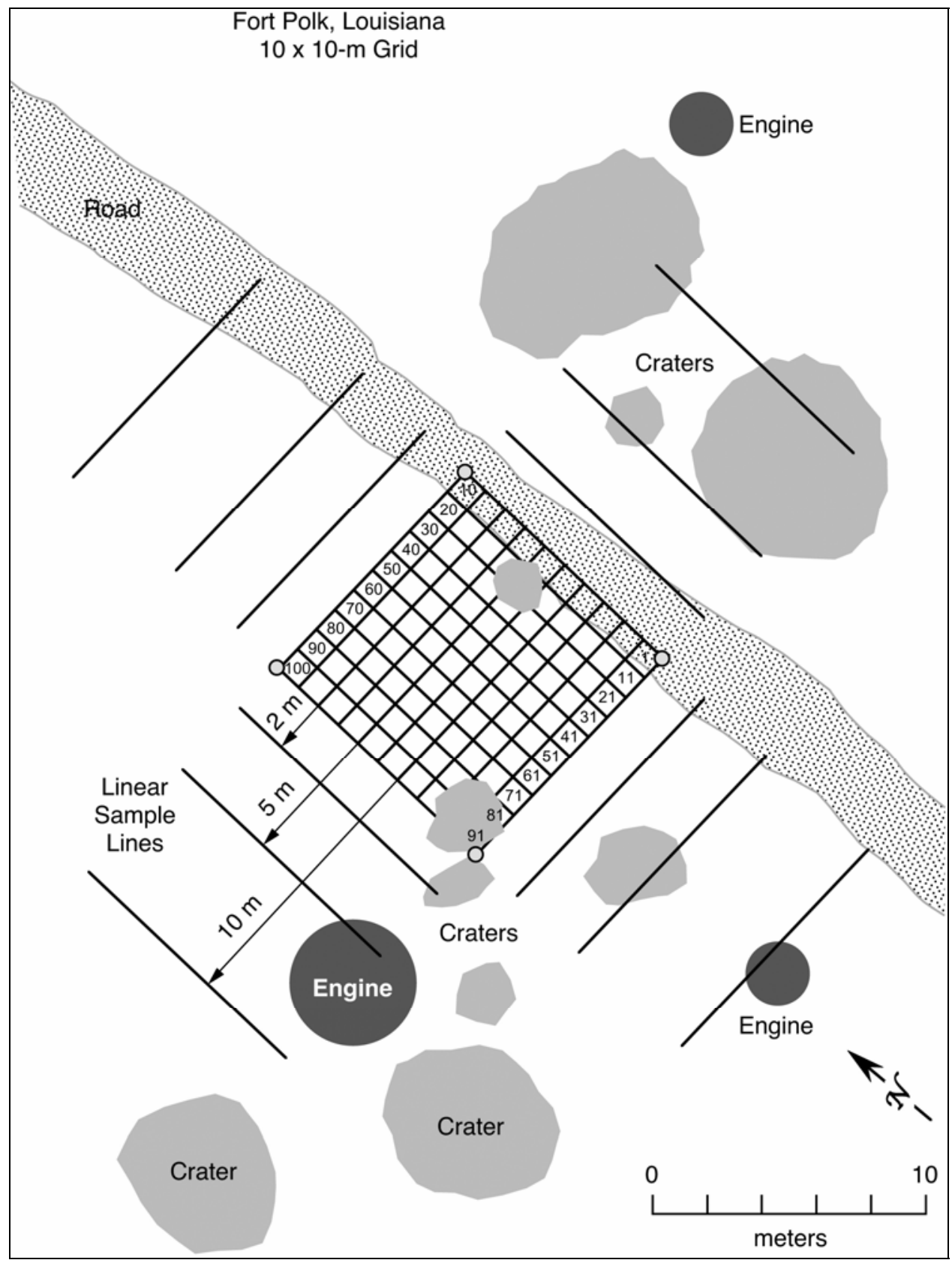

Figure 4. 10-m $\times 10-\mathrm{m}$ sampling grid subdivided into $100-\mathrm{m}^{2}$ minigrids and linear sampling lines around the major grid area. 


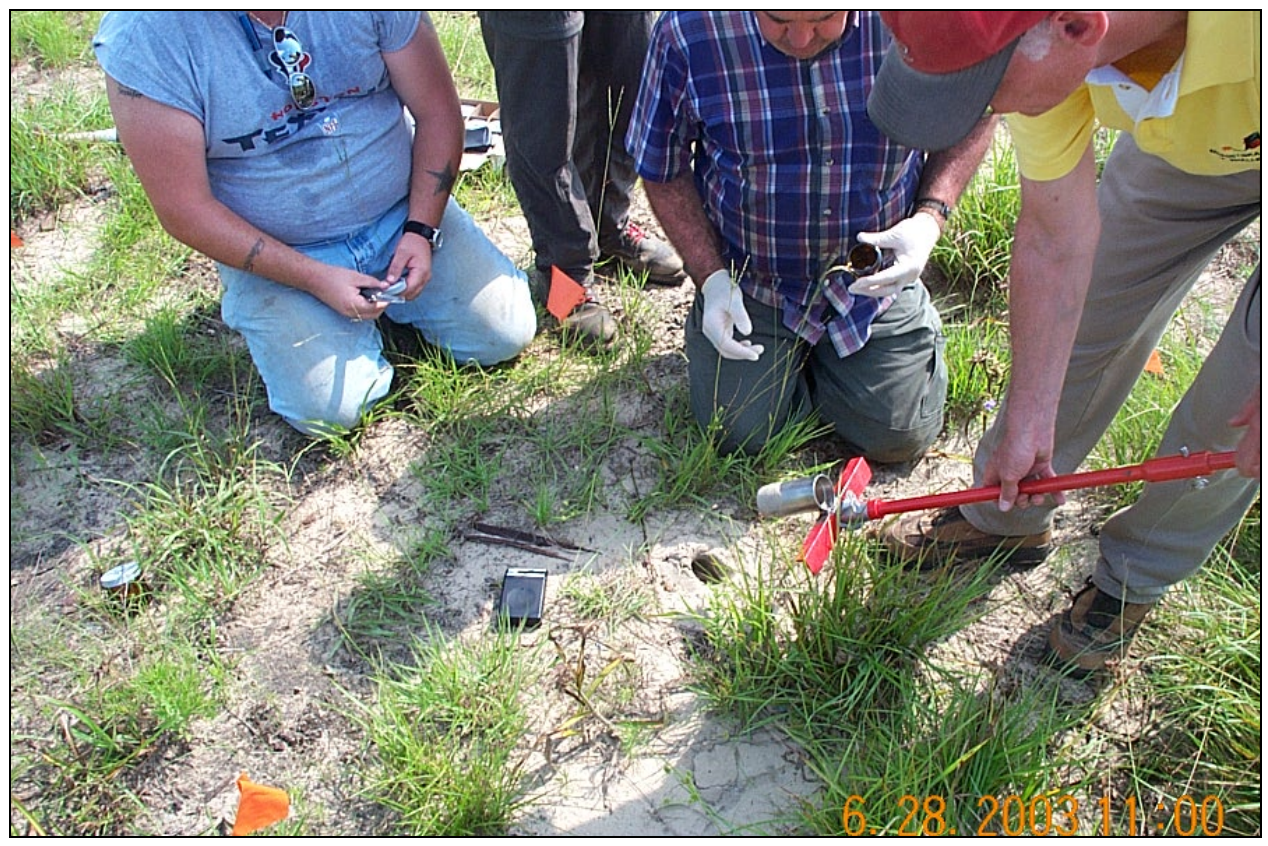

Figure 5. Coring device used to collect soil samples at Fort Polk, Louisiana.

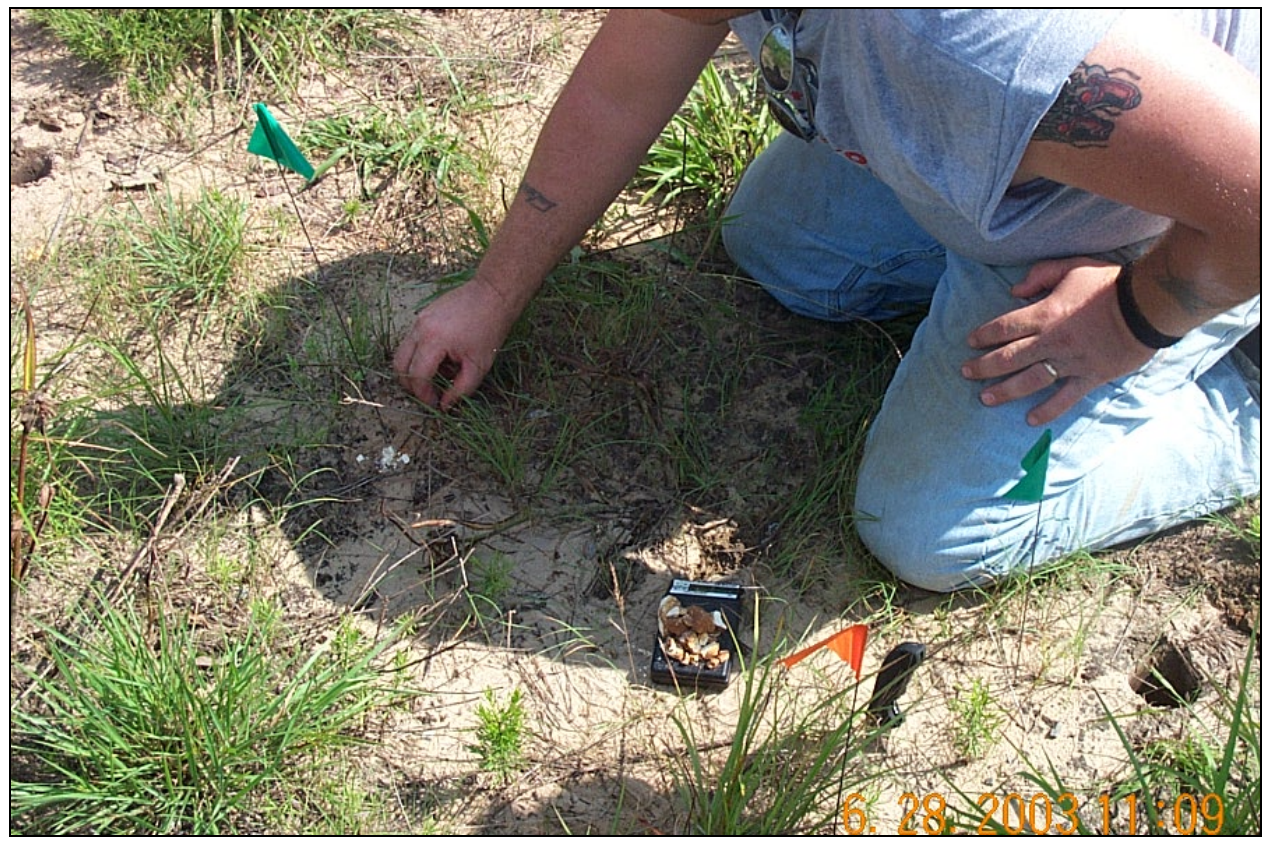

Figure 6. Collecting and weighing visible pieces of Composition B found around a partial detonation of an 81- $\mathrm{mm}$ mortar. 
Composite soil samples were placed on sheets of aluminum foil to air-dry. Dried samples were weighed and sieved though a \#10 sieve. The material that passed the sieve was weighed and ground in a Lab TechEssa LM2 (LabTech Essa Pty. Ltd., Bassendean, WA, Australia) puck mill grinder for 60 seconds. After grinding, composite samples were mixed thoroughly and then spread out to form a 1- to 2-cm-thick layer. A subsample then was obtained by collecting at least 30 increments randomly from the ground material for a mass of about $10 \mathrm{~g}$. For every tenth sample, an additional subsample was collected in an identical manner to enable an assessment of subsampling uncertainty. Each 10-g subsample was extracted with $20 \mathrm{~mL}$ of acetonitrile in an ultrasonic bath overnight at room temperature. After sonication, samples were removed from the bath and allowed to settle for at least an hour. An aliquot was then removed, filtered, and placed in a 7-mL amber vial for storage in a refrigerator.

Commercial sand was used as a laboratory processing blank. This blank soil was ground, subsampled, and extracted with each batch (i.e., approximately 20 samples) of field samples. A standard soil obtained from the U.S. Army Environmental Center was used for preparation of the laboratory control sample. This soil was spiked with a suite of target analytes and was used to assess recovery.

The extracts from both the discrete and composite samples were all analyzed using the general procedures of SW 846 Method 8330 (EPA 1994). For this analysis, an aliquot of each sample was diluted one part extract to three parts reagent-grade water. Analysis was conducted on a modular RP-HPLC system from Thermo Finnigan composed of a SpectraSYSTEM Model P1000 isocratic pump, a SpectraSYSTEM UV2000 dual wavelength UV/VS absorbance detector set at 210 and $254 \mathrm{~nm}$ (cell path $1 \mathrm{~cm}$ ), and a SpectraSYSTEM AS300 autosampler. Samples were introduced by overfilling a $100-\mu \mathrm{L}$ sampling loop. Separations were made on a $15-\mathrm{cm} \times 3.9-\mathrm{mm}(4-\mu \mathrm{m})$ NovaPak C-8 column (Waters Chromatography Division, Milford, Massachusetts) maintained at $28^{\circ} \mathrm{C}$ and eluted with 15:85 isopropanol/water (v/v) at $1.4 \mathrm{~mL} / \mathrm{min}$. Concentrations were estimated from peak heights compared to commercial multianalyte standards (Restek). If concentrations exceeded 20 ppm, an aliquot of the original extract was diluted appropriately with additional acetonitrile prior to the 1 to 4 dilution with reagent-grade water. Estimates of detection limits for the target analytes for this method are given in Table 1 .

For low-concentration $(<0.2 \mathrm{mg} / \mathrm{kg}$ ) samples, a second analysis was conducted by GC-ECD following the general procedure outlined in SW846 Method 8095 (EPA 1999). These analyses were conducted on an HP 6890 Gas Chromatograph equipped with a micro ECD detector. Direct injection of $1 \mu \mathrm{L}$ of soil extract was made into a purged packed inlet port $\left(250^{\circ} \mathrm{C}\right)$ equipped with a 
deactivated Restek Uniliner. Primary separation was conducted on a 6-m- $\times$ 0.53 -mm-ID fused-silica column, with a 1.5- $\mu$ m film thickness of $5 \%$ (phenyl)methylsiloxane (Rtx-5 from Restek, Bellefonte, Pennsylvania).

The GC oven was temperature-programmed as follows: $100^{\circ} \mathrm{C}$ for $2 \mathrm{~min}$, $10^{\circ} \mathrm{C} / \mathrm{min}$ ramp to $280^{\circ} \mathrm{C}$. The carrier gas was hydrogen at $10 \mathrm{~mL} / \mathrm{min}$ (linear velocity approximately $90 \mathrm{~cm} / \mathrm{sec}$ ). The ECD detector temperature was $310^{\circ} \mathrm{C}$ and the makeup gas was nitrogen flowing at $45 \mathrm{~mL} / \mathrm{min}$. If a peak was observed in the retention window for a specific signature compound, the extract was reanalyzed on a confirmation column, $6-\mathrm{m} \times 0.53-\mathrm{mm}$ ID having a $1.5-\mu \mathrm{m}$ film thickness of a proprietary polymer (Rtx-TNT-2 from Restek). The GC oven was temperature-programmed as follows: $130^{\circ} \mathrm{C}$ for $1 \mathrm{~min}, 10^{\circ} \mathrm{C} / \mathrm{min}$ ramp to $280^{\circ} \mathrm{C}$. The carrier gas was helium at $20 \mathrm{~mL} / \mathrm{min}$ (linear velocity approximately 180 $\mathrm{cm} / \mathrm{sec}$ ) and the nitrogen makeup gas was flowing at $60 \mathrm{~mL} / \mathrm{min}$. Inlet and detector temperature were the same as above. Multianalyte standards were purchased from Restek and the instrument was calibrated over five concentrations. Estimates of the detection limits for the GC-ECD method are given in Table 1.

Table 1. Explosives detection limits for soil and water.

\begin{tabular}{|c|c|c|c|}
\hline \multirow{2}{*}{ Analyte } & \multicolumn{2}{|c|}{ Soil $(\boldsymbol{\mu g} / \mathbf{k g})$} & Water $(\boldsymbol{\mu g} / \mathbf{L})$ \\
\cline { 2 - 4 } & RP-HPLC & GC-ECD & GC-ECD \\
\hline HMX & 26 & 10 & 0.047 \\
\hline RDX & 34 & 6 & 0.035 \\
\hline TNB & 16 & 3 & 0.016 \\
\hline TNT & 16 & 2 & 0.017 \\
\hline $2,6-D N T$ & 19 & 2 & 0.009 \\
\hline $2,4-D N T$ & 28 & 2 & 0.010 \\
\hline 2ADNT & 38 & 2 & 0.028 \\
\hline 4ADNT & 32 & 2 & 0.018 \\
\hline NG & 20 & 10 & 0.20 \\
\hline DNA & Co-elutes with NB & 2 & 0.019 \\
\hline DNB & 15 & 2 & 0.010 \\
\hline Tetryl & 100 & 10 & 0.025 \\
\hline PETN & 56 & 16 & 0.24 \\
\hline
\end{tabular}




\section{$4 \quad$ RESULTS}

\section{Quality control}

Results for the analysis of laboratory duplicate soils samples, blank soils, and laboratory control (spike) samples (LCS) are shown in Table 2. No target analytes were detected in any of the blank soils. The recovery for the spiked LCS samples ranged from $83 \%$ to $108 \%$, with a mean value of $97.6 \%$. Eight composite samples had laboratory duplicates removed for analysis. In most cases the results for the laboratory duplicates indicated that the results were very reproducible. For soil sample P-58, however, the agreement of the duplicates was poor, so we examined the sample further. Upon careful inspection it was obvious that the soil sample was not adequately ground because the texture was not uniform. We reground this sample and duplicate subsamples were taken, extracted, and analyzed. The original data for sample P-58 and data for the sample after regrinding are given in Table 2.

\begin{tabular}{|c|c|c|c|c|c|c|c|c|c|}
\hline \multirow[b]{2}{*}{ Lab \# } & \multicolumn{9}{|c|}{ Soil concentration $(\mathrm{mg} / \mathrm{kg})$} \\
\hline & HMX & TNB & RDX & TNT & NG & 2,4-DNT & 2,6-DNT & 4ADNT & 2ADNT \\
\hline \multicolumn{10}{|c|}{ Laboratory duplicate samples } \\
\hline P-58 & 0.162 & $<\mathrm{d}$ & 0.432 & 0.032 & 0.102 & $<\mathrm{d}$ & 0.086 & 0.086 & 0.050 \\
\hline P-58LD & $<d$ & $<d$ & 0.118 & 0.036 & 1.63 & 0.176 & 0.008 & 0.010 & 0.007 \\
\hline \multicolumn{10}{|c|}{ After regrinding P-58 } \\
\hline P-58-1-1 & 0.040 & $<d$ & 0.116 & 0.034 & 0.05 & 0.372 & 0.018 & 0.006 & 0.004 \\
\hline P-58-1-2 & 0.050 & $<d$ & 0.138 & 0.032 & 0.20 & 0.376 & 0.006 & 0.006 & 0.008 \\
\hline $\mathrm{P}-72$ & $<d$ & $<d$ & 0.062 & $<\mathrm{d}$ & $<\mathrm{d}$ & $<\mathrm{d}$ & $<\mathrm{d}$ & $<d$ & $<d$ \\
\hline P-72LD & $<d$ & $<d$ & 0.060 & $<d$ & $<d$ & $<d$ & $<d$ & 0.002 & 0.002 \\
\hline P-80 & 0.614 & $<d$ & 4.56 & 1.20 & $<d$ & $<d$ & $<d$ & 0.158 & 0.156 \\
\hline P-80LD & 0.614 & $<d$ & 4.58 & 1.20 & $<d$ & $<d$ & $<d$ & 0.140 & 0.156 \\
\hline P-89 & 15.1 & $<d$ & 16.4 & 1.21 & $<d$ & $<d$ & $<d$ & 0.246 & 0.328 \\
\hline P-89LD & 15.4 & $<d$ & 15.4 & 1.17 & $<d$ & $<d$ & $<d$ & 0.244 & 0.288 \\
\hline P-98 & 0.224 & $<d$ & 1.23 & 2.24 & $<d$ & $<d$ & $<d$ & 0.612 & 0.890 \\
\hline P-98LD & 0.226 & $<d$ & 1.22 & 2.20 & $<d$ & $<d$ & $<\mathrm{d}$ & 0.610 & 0.860 \\
\hline D-39C & 0.064 & $<d$ & 0.512 & 0.008 & $<d$ & $<d$ & $<d$ & 0.040 & 0.048 \\
\hline D-39C LD & 0.062 & $<d$ & 0.532 & 0.010 & $<d$ & $<d$ & $<d$ & 0.038 & 0.040 \\
\hline
\end{tabular}




\begin{tabular}{|c|c|c|c|c|c|c|c|c|c|}
\hline \multicolumn{10}{|c|}{ Table 2 (cont'd). } \\
\hline \multirow[b]{2}{*}{ Lab \# } & \multicolumn{9}{|c|}{ Soil concentration $(\mathrm{mg} / \mathrm{kg})$} \\
\hline & HMX & TNB & RDX & TNT & NG & 2,4-DNT & 2,6-DNT & 4ADNT & 2ADNT \\
\hline D-82C & 0.674 & $<\mathrm{d}$ & 4.46 & 0.462 & $<\mathrm{d}$ & $<\mathrm{d}$ & $<\mathrm{d}$ & 0.306 & 0.316 \\
\hline D-82C LD & 0.714 & $<d$ & 4.54 & 0.474 & $<d$ & $<\mathrm{d}$ & $<d$ & 0.344 & 0.338 \\
\hline G-1-6 & 8.58 & $<\mathrm{d}$ & 80.0 & 21.2 & $<\mathrm{d}$ & $<\mathrm{d}$ & $<d$ & 0.808 & 0.798 \\
\hline G-1-6 LD & 7.96 & $<\mathrm{d}$ & 75.8 & 20.4 & $<\mathrm{d}$ & $<\mathrm{d}$ & $<\mathrm{d}$ & 0.728 & 0.784 \\
\hline \multicolumn{10}{|l|}{ Lab blanks } \\
\hline LB-1 & $<\mathrm{d}$ & $<d$ & $<d$ & $<\mathrm{d}$ & $<d$ & $<\mathrm{d}$ & $<\mathrm{d}$ & $<\mathrm{d}$ & $<d$ \\
\hline LB-2 & $<d$ & $<d$ & $<d$ & $<\mathrm{d}$ & $<\mathrm{d}$ & $<\mathrm{d}$ & $<\mathrm{d}$ & $<\mathrm{d}$ & $<d$ \\
\hline LB-3 & $<\mathrm{d}$ & $<d$ & $<d$ & $<\mathrm{d}$ & $<\mathrm{d}$ & $<\mathrm{d}$ & $<d$ & $<\mathrm{d}$ & $<d$ \\
\hline LB-4 & $<d$ & $<d$ & $<d$ & $<\mathrm{d}$ & $<\mathrm{d}$ & $<\mathrm{d}$ & $<d$ & $<\mathrm{d}$ & $<d$ \\
\hline LB-5 & $<d$ & $<d$ & $<\mathrm{d}$ & $<\mathrm{d}$ & $<\mathrm{d}$ & $<\mathrm{d}$ & $<d$ & $<\mathrm{d}$ & $<\mathrm{d}$ \\
\hline LB-6 & $<\mathrm{d}$ & $<\mathrm{d}$ & $<\mathrm{d}$ & $<\mathrm{d}$ & $<\mathrm{d}$ & $<\mathrm{d}$ & $<d$ & $<\mathrm{d}$ & $<\mathrm{d}$ \\
\hline \multicolumn{10}{|c|}{ Spike samples } \\
\hline LCS-1 & 0.542 & 0.548 & 0.505 & 0.445 & - & 0.509 & 0.445 & 0.539 & 0.534 \\
\hline LCS-2 & 0.488 & 0.488 & 0.443 & 0.415 & - & 0.475 & 0.477 & 0.482 & 0.463 \\
\hline \multicolumn{10}{|c|}{ Percent recoveries } \\
\hline LCS-1 & $108 \%$ & $110 \%$ & $101 \%$ & $89 \%$ & - & $102 \%$ & $89 \%$ & $108 \%$ & $107 \%$ \\
\hline LCS-2* & $98 \%$ & $98 \%$ & $89 \%$ & $83 \%$ & - & $95.0 \%$ & $95.4 \%$ & $96.4 \%$ & $92.6 \%$ \\
\hline
\end{tabular}

After regrinding, the agreement of the data for all analytes is much improved, confirming that the initial problem with this soil was due to inadequate grinding, most likely because initially the grinding bowl contained too much soil. Subsequently, the laboratory protocol was changed to grinding aliquots of no more than $500 \mathrm{~g}$ (the manufacturer-recommended cutoff is $800 \mathrm{~g}$ ).

Because we were concerned that other soil samples from Fort Polk also may have suffered this problem, we inspected all the composite samples and found five that also appeared to be inadequately ground. These samples also were reground and triplicate subsamples were analyzed. The original results for these samples and the results for the triplicate reground samples are given in Table 3. For these samples, changes in the analytical results were much smaller and often insignificant (i.e., < 15\% RPD). 
Table 3. Analytical results for Fort Polk samples that were analyzed, reground, and subsamples analyzed in triplicate.

\begin{tabular}{|c|c|c|c|c|c|}
\hline \multirow[b]{2}{*}{ Lab \# } & \multicolumn{5}{|c|}{ Soil concentration (mg/kg) } \\
\hline & HMX & RDX & TNT & 4ADNT & 2ADNT \\
\hline $\begin{array}{l}\mathrm{D}-14 \mathrm{C} \\
\text { regrinding }\end{array}$ & 18.5 & 139 & 15.5 & 2.24 & 2.88 \\
\hline 1 & 17.7 & 127 & 14.7 & 2.20 & 2.66 \\
\hline 2 & 17.7 & 128 & 14.9 & 2.14 & 2.72 \\
\hline 3 & 17.5 & 126 & 14.4 & 2.08 & 2.56 \\
\hline $\begin{array}{l}D-46 C \\
\text { regrinding }\end{array}$ & 0.294 & 1.85 & 0.158 & 0.098 & 0.124 \\
\hline 1 & 0.320 & 1.94 & 0.242 & 0.070 & 0.142 \\
\hline 2 & 0.316 & 1.91 & 0.206 & 0.064 & 0.122 \\
\hline 3 & 0.308 & 1.86 & 0.198 & 0.084 & 0.114 \\
\hline $\begin{array}{l}D-51 C \\
\text { regrinding }\end{array}$ & 3.04 & 21.6 & 2.68 & 0.640 & 0.460 \\
\hline 1 & 3.20 & 22.6 & 2.78 & 0.606 & 0.648 \\
\hline 2 & 3.24 & 22.4 & 2.80 & 0.562 & 0.658 \\
\hline 3 & 3.26 & 22.8 & 2.70 & 0.588 & 0.636 \\
\hline D-82C & 0.674 & 4.46 & 0.462 & 0.306 & 0.316 \\
\hline $\begin{array}{l}\text { D-82C LD } \\
\text { regrinding }\end{array}$ & 0.714 & 4.54 & 0.474 & 0.344 & 0.338 \\
\hline 1 & 0.666 & 4.18 & 0.494 & 0.276 & 0.266 \\
\hline 2 & 0.656 & 4.20 & 0.486 & 0.270 & 0.258 \\
\hline 3 & 0.678 & 4.24 & 0.506 & 0.278 & 0.258 \\
\hline $\begin{array}{l}\mathrm{D}-87 \mathrm{C} \\
\text { regrinding }\end{array}$ & 0.228 & 1.504 & 37.4 & 0.278 & 0.260 \\
\hline 1 & 0.220 & 1.468 & 35.2 & 0.260 & 0.230 \\
\hline 2 & 0.218 & 1.422 & 34.2 & 0.272 & 0.228 \\
\hline 3 & 0.240 & 1.428 & 34.2 & 0.264 & 0.226 \\
\hline
\end{tabular}

\section{Grid samples from the area near a low-order 81-mm mortar detonation}

Analytical results for the 100 discrete minigrid samples collected from within a $10-\mathrm{m} \times 10$-m grid from the area where an 81-mm mortar round had lowordered are presented in Table 4. RDX, HMX, TNT, 2ADNT, and 4ADNT were detected in nearly all of the discrete surface soil samples. RDX was present at the highest concentration, with surface soil concentrations ranging over almost five orders of magnitude from 0.037 to $2,390 \mathrm{mg} / \mathrm{kg}$. The median RDX concentration 
was $1.79 \mathrm{mg} / \mathrm{kg}$, but because there were several very high concentrations, mean concentration was $70.9 \mathrm{mg} / \mathrm{kg}$. HMX concentrations in these discrete minigrid samples ranged from less than detection limits $(0.01 \mathrm{mg} / \mathrm{kg})$ to $253 \mathrm{mg} / \mathrm{kg}$. The median ratio of HMX to RDX was 0.176 , which is slightly higher than expected (about 0.11) for HMX as an impurity in RDX. This ratio suggests that weathering has resulted in the preferential dissolution of the more soluble RDX.

Table 4. Results from the analysis of discrete samples from $1001-\mathrm{m} \times 1-\mathrm{m}$ minigrids in an area near a low-order 81-mm mortar detonation at the impact range at Fort Polk. (Analysis by RP-HPLC and GC-ECD [shaded].)

\begin{tabular}{|c|c|c|c|c|c|c|c|c|c|}
\hline \multirow[b]{2}{*}{ Field \# } & \multirow{2}{*}{$\begin{array}{l}\text { Comp. B } \\
\text { found } \\
\text { (g) }\end{array}$} & \multicolumn{5}{|c|}{ Soil concentration $(\mathrm{mg} / \mathrm{kg})$} & \multicolumn{3}{|c|}{ Ratio } \\
\hline & & HMX & RDX & TNT & 4ADNT & 2ADNT & TNT/RDX & HMX/RDX & $\begin{array}{l}\text { 2ADNTI } \\
\text { 4ADNT }\end{array}$ \\
\hline D1 & & 0.893 & 8.86 & 2.72 & 0.124 & 0.170 & 0.307 & 0.101 & 1.38 \\
\hline D2 & 0.1 & 0.639 & 3.50 & 0.071 & 0.178 & 0.198 & 0.020 & 0.182 & 1.11 \\
\hline D3 & 50.2 & 1.12 & 5.02 & 0.072 & 0.563 & 0.590 & 0.014 & 0.223 & 1.05 \\
\hline D4 & 0.1 & 7.50 & 42.7 & 6.53 & 0.418 & 0.410 & 0.153 & 0.176 & 0.98 \\
\hline D5 & 0.1 & 44.4 & 385 & 147 & 3.18 & 3.10 & 0.382 & 0.115 & 0.97 \\
\hline D6 & 0.3 & 2.46 & 24.9 & 0.095 & 0.707 & 0.715 & 0.004 & 0.099 & 1.01 \\
\hline D7 & & 0.740 & 3.64 & 0.064 & 0.115 & 0.125 & 0.018 & 0.203 & 1.10 \\
\hline D8 & & 0.190 & 0.965 & 0.003 & 0.047 & 0.068 & 0.004 & 0.197 & 1.44 \\
\hline D9 & & 0.126 & 0.526 & 0.002 & 0.028 & 0.029 & 0.004 & 0.239 & 1.06 \\
\hline D10 & & 0.041 & 0.161 & 0.004 & 0.009 & 0.008 & 0.026 & 0.252 & 0.89 \\
\hline D11 & & 0.230 & 1.18 & 0.003 & 0.099 & 0.129 & 0.003 & 0.195 & 1.30 \\
\hline D12 & & 0.235 & 1.03 & 0.023 & 0.510 & 0.633 & 0.023 & 0.227 & 1.24 \\
\hline D13 & 28.7 & 11.3 & 64.3 & 13.4 & 1.32 & 1.75 & 0.208 & 0.176 & 1.33 \\
\hline D14 & 7.8 & 60.2 & 557 & 164 & 3.30 & 3.61 & 0.294 & 0.108 & 1.09 \\
\hline D15 & 5.5 & 189 & 1790 & 489 & 16.3 & 15.3 & 0.273 & 0.106 & 0.94 \\
\hline D16 & 17 & 253 & 2390 & 1560 & 0.090 & 0.125 & 0.653 & 0.106 & 1.38 \\
\hline D17 & 0.3 & 1.27 & 11.3 & 2.50 & 0.392 & 0.344 & 0.222 & 0.113 & 0.88 \\
\hline D18 & & 0.371 & 1.65 & 0.084 & 0.265 & 0.244 & 0.051 & 0.224 & 0.92 \\
\hline D19 & & 0.075 & 0.335 & 0.024 & 0.036 & 0.042 & 0.070 & 0.225 & 1.16 \\
\hline D20 & & 0.028 & 0.263 & $0.001^{*}$ & 0.009 & 0.011 & 0.004 & 0.108 & 1.20 \\
\hline D21 & 1.4 & 5.74 & 48.3 & 29.7 & 0.349 & 0.476 & 0.615 & 0.119 & 1.36 \\
\hline D22 & 0.2 & 1.42 & 13.3 & 4.46 & 0.366 & 0.457 & 0.334 & 0.106 & 1.25 \\
\hline D23 & & 0.484 & 3.36 & 0.901 & 0.622 & 0.706 & 0.268 & 0.144 & 1.13 \\
\hline D24 & 3 & 0.925 & 6.93 & 0.240 & 1.50 & 1.64 & 0.035 & 0.133 & 1.10 \\
\hline D25 & 48.2 & 97.4 & 889 & 321 & 5.90 & 5.47 & 0.362 & 0.110 & 0.93 \\
\hline D26 & 13.1 & 3.56 & 21.8 & 0.932 & 1.76 & 1.91 & 0.043 & 0.164 & 1.09 \\
\hline
\end{tabular}


Table 4 (cont'd). Results from the analysis of discrete samples from $1001-\mathrm{m} \times 1-\mathrm{m}$ minigrids in an area near a low-order 81-mm mortar detonation at the impact range at Fort Polk. (Analysis by RP-HPLC and GC-ECD [shaded].)

\begin{tabular}{|c|c|c|c|c|c|c|c|c|c|}
\hline \multirow[b]{2}{*}{ Field \# } & \multirow{2}{*}{$\begin{array}{l}\text { Comp. B } \\
\text { found } \\
\text { (g) }\end{array}$} & \multicolumn{5}{|c|}{ Soil concentration (mg/kg) } & \multicolumn{3}{|c|}{ Ratio } \\
\hline & & HMX & RDX & TNT & 4ADNT & 2ADNT & TNT/RDX & HMX/RDX & $\begin{array}{l}\text { 2ADNTI } \\
\text { 4ADNT }\end{array}$ \\
\hline D27 & 1 & 0.528 & 3.75 & 0.022 & 0.153 & 0.182 & 0.006 & 0.141 & 1.19 \\
\hline D28 & 0.4 & 0.185 & 0.618 & 0.002 & 0.038 & 0.045 & 0.003 & 0.299 & 1.17 \\
\hline D29 & & 0.077 & 0.193 & 0.004 & 0.034 & 0.040 & 0.022 & 0.402 & 1.19 \\
\hline D30 & & 0.016 & 0.081 & $0.001^{*}$ & 0.008 & 0.009 & 0.012 & 0.198 & 1.13 \\
\hline D31 & 0.4 & 0.419 & 1.65 & 0.003 & 0.195 & 0.253 & 0.002 & 0.255 & 1.30 \\
\hline D32 & & 0.561 & 1.56 & 0.017 & 0.394 & 0.456 & 0.011 & 0.358 & 1.16 \\
\hline D33 & & 0.476 & 8.51 & 0.247 & 0.710 & 0.636 & 0.029 & 0.056 & 0.89 \\
\hline D34 & & 1.42 & 10.6 & 3.47 & 0.566 & 0.691 & 0.329 & 0.135 & 1.22 \\
\hline D35 & & 0.215 & 2.24 & 0.106 & 0.878 & 1.03 & 0.047 & 0.096 & 1.17 \\
\hline D36 & & 3.35 & 25.2 & 6.88 & 1.04 & 1.13 & 0.273 & 0.133 & 1.08 \\
\hline D37 & & 2.04 & 7.15 & 0.168 & 0.590 & 0.698 & 0.023 & 0.285 & 1.18 \\
\hline D38 & & 0.082 & 0.248 & 0.003 & 0.038 & 0.051 & 0.014 & 0.332 & 1.36 \\
\hline D39 & & 0.067 & 0.175 & 0.009 & 0.049 & 0.053 & 0.049 & 0.385 & 1.08 \\
\hline D40 & & $0.005^{\star}$ & 0.037 & $0.001^{*}$ & 0.009 & 0.013 & 0.027 & 0.135 & 1.50 \\
\hline D41 & & 2.17 & 7.52 & 0.871 & 0.848 & 0.910 & 0.116 & 0.289 & 1.07 \\
\hline D42 & & 1.66 & 5.65 & 0.132 & 0.586 & 0.777 & 0.023 & 0.294 & 1.33 \\
\hline D43 & & 0.304 & 1.97 & 0.027 & 0.342 & 0.348 & 0.014 & 0.154 & 1.02 \\
\hline D44 & 0.1 & 0.108 & 0.571 & 0.009 & 0.347 & 0.471 & 0.016 & 0.189 & 1.36 \\
\hline D45 & 0.1 & 1.76 & 4.84 & 0.062 & 0.461 & 0.510 & 0.013 & 0.365 & 1.11 \\
\hline D46 & & 2.52 & 19.9 & 3.94 & 0.711 & 0.668 & 0.198 & 0.126 & 0.94 \\
\hline D47 & & 0.232 & 0.825 & 0.014 & 0.098 & 0.134 & 0.017 & 0.281 & 1.36 \\
\hline D48 & & 0.036 & 0.122 & 0.004 & 0.020 & 0.027 & 0.031 & 0.296 & 1.38 \\
\hline D49 & & 0.092 & 1.46 & 0.004 & 0.015 & 0.018 & 0.002 & 0.063 & 1.18 \\
\hline D50 & & 0.020 & 0.070 & $0.001^{*}$ & 0.010 & 0.010 & 0.014 & 0.292 & 1.00 \\
\hline D51 & 0.2 & 34.9 & 331 & 81.6 & 2.23 & 2.15 & 0.246 & 0.105 & 0.96 \\
\hline D52 & & 1.88 & 9.70 & 0.189 & 0.350 & 0.488 & 0.019 & 0.194 & 1.39 \\
\hline D53 & 16.3 & 1.54 & 3.96 & 0.434 & 0.915 & 1.17 & 0.110 & 0.390 & 1.28 \\
\hline D54 & & 0.725 & 1.44 & 0.014 & 0.253 & 0.246 & 0.010 & 0.504 & 0.97 \\
\hline D55 & & 0.517 & 3.67 & 1.31 & 0.108 & 0.104 & 0.356 & 0.141 & 0.96 \\
\hline D56 & & 0.043 & 0.243 & 0.001 & 0.023 & 0.027 & 0.004 & 0.176 & 1.17 \\
\hline D57 & & 0.669 & 3.21 & 0.005 & 0.037 & 0.068 & 0.002 & 0.208 & 1.82 \\
\hline D58 & & 0.094 & 0.254 & 0.006 & 0.035 & 0.054 & 0.023 & 0.369 & 1.53 \\
\hline D59 & & 0.086 & 1.03 & 0.012 & 0.028 & 0.027 & 0.011 & 0.083 & 0.95 \\
\hline D60 & & $0.005^{\star}$ & 0.073 & $0.001^{*}$ & 0.012 & 0.016 & 0.014 & 0.069 & 1.38 \\
\hline
\end{tabular}




\begin{tabular}{|c|c|c|c|c|c|c|c|c|c|}
\hline \multicolumn{10}{|c|}{ Table 4 (cont'd). } \\
\hline \multirow[b]{2}{*}{ Field \# } & \multirow{2}{*}{$\begin{array}{l}\text { Comp. B } \\
\text { found } \\
\text { (g) }\end{array}$} & \multicolumn{5}{|c|}{ Soil concentration $(\mathrm{mg} / \mathrm{kg})$} & \multicolumn{3}{|c|}{ Ratio } \\
\hline & & HMX & RDX & TNT & 4ADNT & 2ADNT & TNT/RDX & HMX/RDX & $\begin{array}{l}\text { 2ADNTI } \\
\text { 4ADNT }\end{array}$ \\
\hline D61 & & 2.55 & 12.7 & 0.924 & 1.41 & 1.76 & 0.073 & 0.201 & 1.25 \\
\hline D62 & 1.1 & 16.5 & 138 & 76.1 & 3.14 & 3.76 & 0.552 & 0.120 & 1.20 \\
\hline D63 & 0.9 & 6.38 & 53.7 & 28.2 & 0.919 & 1.03 & 0.525 & 0.119 & 1.12 \\
\hline D64 & 0.5 & 0.736 & 3.85 & 0.028 & 0.893 & 1.15 & 0.007 & 0.191 & 1.29 \\
\hline D65 & 1.2 & 0.585 & 4.94 & 0.656 & 0.156 & 0.176 & 0.133 & 0.119 & 1.13 \\
\hline D66 & 0.1 & 0.269 & 1.22 & 0.007 & 0.130 & 0.168 & 0.005 & 0.221 & 1.29 \\
\hline D67 & & 0.535 & 4.63 & 1.70 & 0.063 & 0.079 & 0.368 & 0.115 & 1.25 \\
\hline D68 & & 0.117 & 0.470 & 0.003 & 0.013 & 0.022 & 0.006 & 0.248 & 1.70 \\
\hline D69 & & 0.188 & 2.41 & 0.007 & 0.023 & 0.025 & 0.003 & 0.078 & 1.07 \\
\hline D70 & & 0.216 & 1.06 & 0.044 & 0.063 & 0.173 & 0.041 & 0.203 & 2.75 \\
\hline D71 & & 5.91 & 30.8 & 5.41 & 0.486 & 0.515 & 0.176 & 0.192 & 1.06 \\
\hline D72 & & 0.468 & 1.40 & 0.054 & 0.755 & 0.813 & 0.039 & 0.333 & 1.08 \\
\hline D73 & & 2.23 & 12.5 & 1.32 & 0.619 & 0.736 & 0.106 & 0.179 & 1.19 \\
\hline D74 & & 0.044 & 0.342 & 0.006 & 0.117 & 0.173 & 0.016 & 0.128 & 1.48 \\
\hline D75 & & $0.005^{\star}$ & 0.074 & 0.002 & 0.019 & 0.025 & 0.021 & 0.067 & 1.33 \\
\hline D76 & & 0.237 & 1.11 & 0.007 & 0.047 & 0.086 & 0.007 & 0.212 & 1.84 \\
\hline D77 & & 0.109 & 0.180 & $0.001^{*}$ & 0.016 & 0.022 & 0.006 & 0.605 & 1.36 \\
\hline D78 & & 0.022 & 0.076 & $0.001^{*}$ & 0.015 & 0.018 & 0.013 & 0.286 & 1.25 \\
\hline D79 & & 0.667 & 7.11 & 2.58 & 0.087 & 0.076 & 0.363 & 0.094 & 0.87 \\
\hline D80 & & 0.058 & 0.187 & 0.004 & 0.017 & 0.031 & 0.023 & 0.311 & 1.83 \\
\hline D81 & & 0.089 & 0.805 & 0.045 & 0.238 & 0.235 & 0.056 & 0.110 & 0.99 \\
\hline D82 & & 2.80 & 24.1 & 3.29 & 1.11 & 1.09 & 0.136 & 0.116 & 0.98 \\
\hline D83 & & 1.55 & 7.73 & 0.783 & 0.479 & 0.550 & 0.101 & 0.201 & 1.15 \\
\hline D84 & & 0.098 & 0.539 & 0.003 & 0.102 & 0.129 & 0.005 & 0.182 & 1.26 \\
\hline D85 & & 0.095 & 0.260 & 0.003 & 0.045 & 0.045 & 0.010 & 0.364 & 1.00 \\
\hline D86 & & 0.031 & 0.233 & $0.001^{*}$ & 0.018 & 0.021 & 0.004 & 0.134 & 1.15 \\
\hline D87 & & 0.076 & 0.366 & 0.003 & 0.021 & 0.049 & 0.009 & 0.208 & 2.38 \\
\hline D88 & & 0.225 & 1.93 & 0.396 & 0.075 & 0.069 & 0.205 & 0.116 & 0.92 \\
\hline D89 & & 0.083 & 0.731 & 0.002 & 0.012 & 0.026 & 0.003 & 0.114 & 2.17 \\
\hline D90 & & 0.108 & 0.138 & 0.004 & 0.022 & 0.022 & 0.031 & 0.781 & 1.00 \\
\hline D91 & & 1.57 & 17.1 & 1.68 & 0.739 & 0.769 & 0.098 & 0.092 & 1.04 \\
\hline D92 & & 0.358 & 1.27 & 0.093 & 0.095 & 0.126 & 0.073 & 0.281 & 1.32 \\
\hline D93 & & 0.065 & 0.829 & 0.038 & 0.154 & 0.157 & 0.046 & 0.078 & 1.02 \\
\hline D94 & & 0.111 & 0.908 & 0.232 & 0.031 & 0.052 & 0.256 & 0.122 & 1.68 \\
\hline D95 & & 0.777 & 10.9 & 0.015 & 0.103 & 0.142 & 0.001 & 0.071 & 1.38 \\
\hline D96 & & 0.516 & 4.44 & 1.86 & 0.054 & 0.109 & 0.419 & 0.116 & 2.00 \\
\hline
\end{tabular}


Table 4 (cont'd). Results from the analysis of discrete samples from $1001-\mathrm{m} \times 1-\mathrm{m}$ minigrids in an area near a low-order 81-mm mortar detonation at the impact range at Fort Polk. (Analysis by RP-HPLC and GC-ECD [shaded].)

\begin{tabular}{|c|c|c|c|c|c|c|c|c|c|}
\hline & \multirow{2}{*}{$\begin{array}{c}\text { Comp. B } \\
\text { found } \\
\text { Field \# }\end{array}$} & \multicolumn{9}{|c|}{ Soil concentration (mg/kg) } & \multicolumn{3}{c|}{ Ratio } \\
\cline { 3 - 11 } & HMX & RDX & TNT & 4ADNT & 2ADNT & TNT/RDX & HMX/RDX & $\begin{array}{c}\text { 2ADNTI } \\
\text { 4ADNT }\end{array}$ \\
\hline D97 & & 0.059 & 0.437 & 0.102 & 0.019 & 0.034 & 0.232 & 0.135 & 1.81 \\
\hline D98 & & 0.047 & 0.354 & 0.003 & 0.024 & 0.038 & 0.009 & 0.134 & 1.60 \\
\hline D99 & & 0.170 & 1.52 & $0.001^{*}$ & 0.016 & 0.020 & 0.001 & 0.112 & 1.23 \\
\hline D100 & & $0.005^{\star}$ & 0.067 & $0.001^{\star}$ & 0.013 & 0.022 & 0.015 & 0.074 & 1.60 \\
\hline Max & & 253 & 2390 & 1560 & 16.3 & 15.3 & 0.653 & 0.781 & 2.75 \\
\hline Min & & 0.005 & 0.037 & 0.001 & 0.008 & 0.008 & 0.001 & 0.056 & 0.873 \\
\hline Median & & 0.395 & 1.79 & 0.044 & 0.120 & 0.169 & 0.025 & 0.176 & 1.18 \\
\hline Mean & & 7.89 & 70.9 & 29.7 & 0.626 & 0.663 & 0.107 & 0.195 & 1.25 \\
\hline
\end{tabular}

* Concentration was nondetectable; one-half the detection limit was used for mathematical computation.

Note: Because the distributions depart greatly from normal as shown by the large discrepancy between median and mean and by the histogram, it is not possible to compute useful standard deviations.

TNT concentrations in these samples were always lower than RDX and ranged from less than $0.002 \mathrm{mg} / \mathrm{kg}$ to $1,560 \mathrm{mg} / \mathrm{kg}$ (Table 4). The mean ratio of TNT to RDX was 0.107. For non-weathered Composition B, the ratio should be about 0.7 , indicating that the TNT present in these samples has been subject to preferential dissolution and environmental transformation. Even so, based on our experience, the presence of TNT in these samples is consistent with the contamination source being Composition B.

The two most common environmental transformation products of TNT, 2ADNT and 4ADNT, were detected in all 100 minigrid samples, even in samples where the TNT concentration was below detection limits. In fact, the median concentrations of these two transformation products were actually higher than TNT for these 100 samples. The median ratio of 2ADNT/4ADNT was 1.18 and, from our experience, this ratio is typical for these compounds in surface soil samples.

The distribution of the 100 discrete minigrid concentrations for RDX is presented as a histogram in Figure 7; the distribution is clearly non-Gaussian. A histogram presenting the log of the concentration versus frequency is shown in Figure 8. 


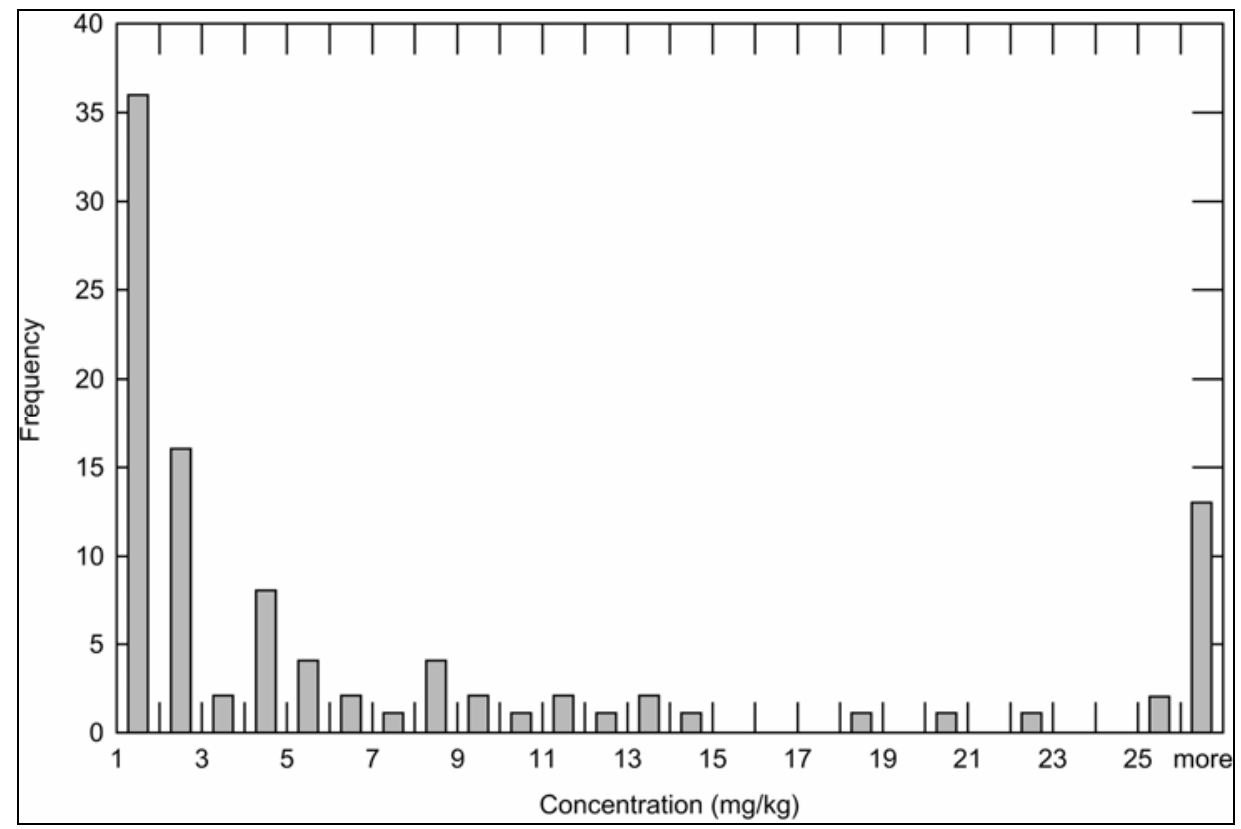

Figure 7. Distribution of soil RDX concentrations from 100 discrete soil samples taken in the 1-m $\times 1-m$ minigrids.

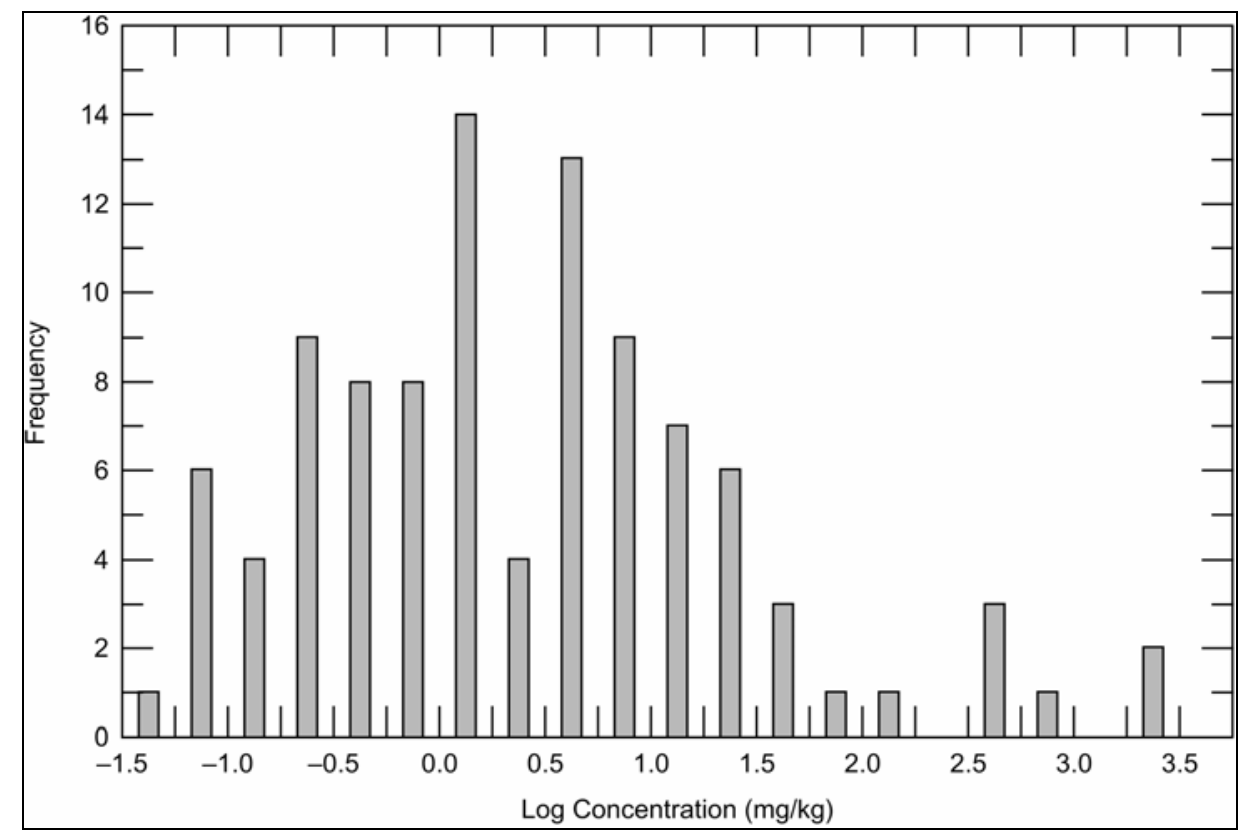

Figure 8. Distribution of the log soil RDX concentrations from 100 discrete soil samples taken from 1-m $\times 1-\mathrm{m}$ minigrids. 


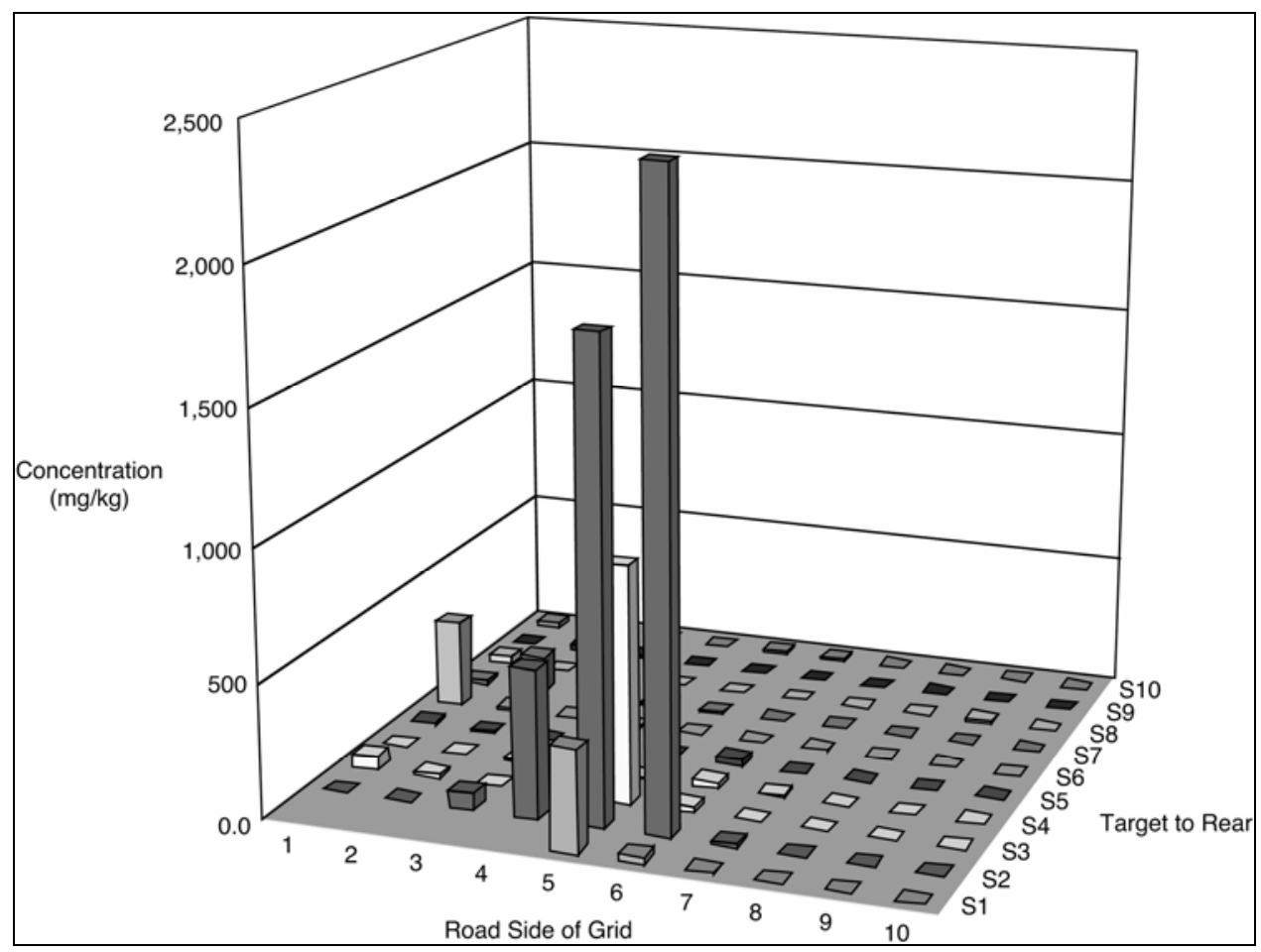

Figure 9. Soil concentration of RDX relative to its location within the $10-\mathrm{m} \times$ 10-m grid.

A plot of the RDX concentrations in surface soil versus position within the $10-\mathrm{m} \times 10$-m grid is presented in Figure 9. It appears from these data that one major hot spot within this grid is centered at minigrid D15 and a smaller one near the edge of the grid is centered at grid D62. These apparent hot spots can also be seen in the weights of Composition B recovered from the individual minigrids (Table 4), although the centers of the hot spots do not agree exactly (Figure 10). These hot spots coincide with the high concentration population in Figure 8 and are spatially distinct. The lack of complete agreement shows that the presence of visible residues on the surface is not necessarily a prerequisite to finding high concentrations in the soil, i.e., there can be areas adjacent to visible residues with high concentrations of fine non-visible particles. 


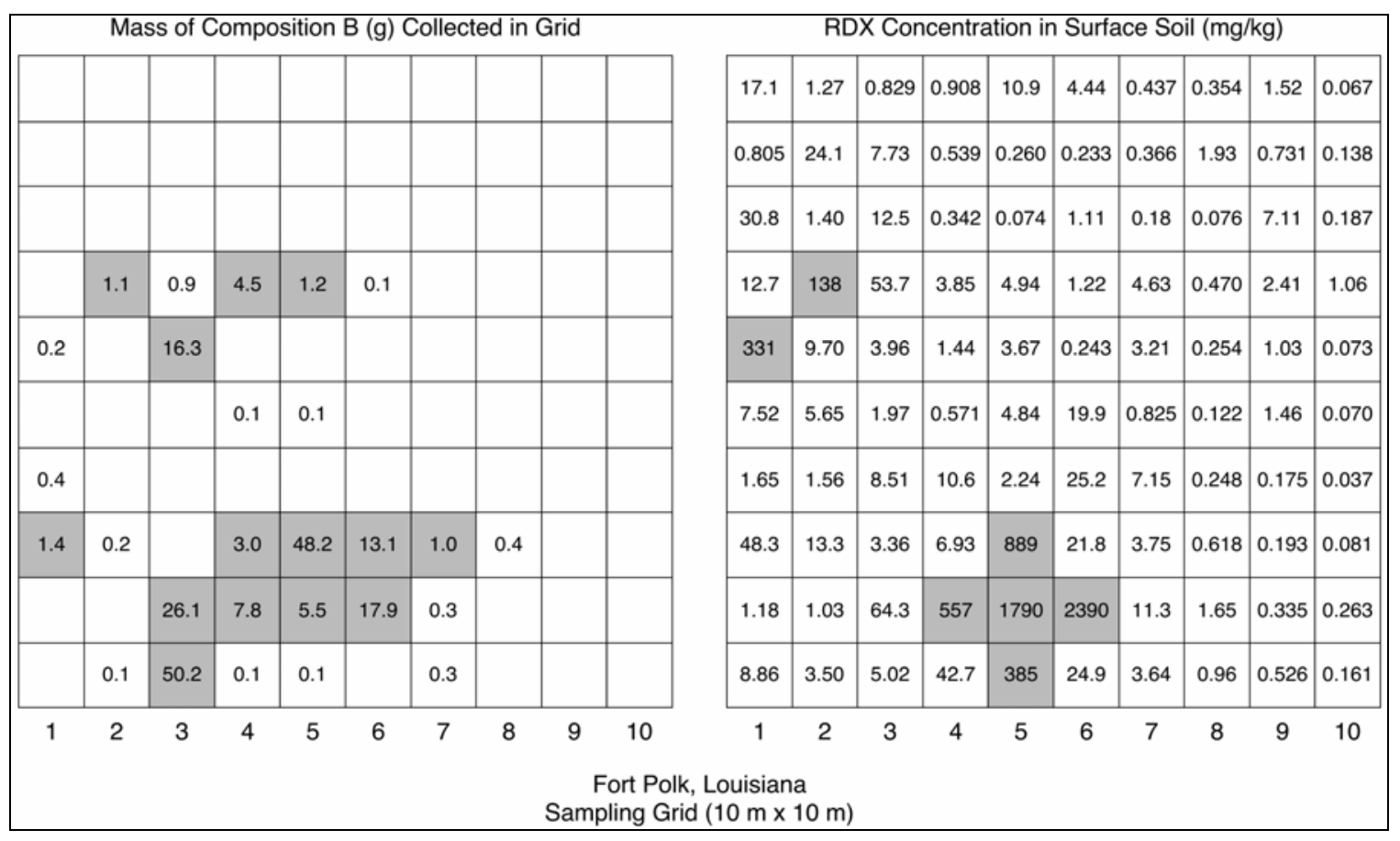

Figure 10. Weight of Composition B and soil RDX concentration and their relative position in the sampling grid.

\section{Comparison of field duplicate discrete and ten-increment composites for minigrid samples}

Results for the duplicate discrete samples from the 20 randomly selected minigrids are presented in Table 5. An inspection of these data indicates that agreement between replicates is analyte-dependant. For example, while there is greater than a two-orders-of-magnitude difference between the RDX, TNT, and HMX concentrations for the field duplicates taken from minigrids 80 and 100, the discrepancy between 4ADNT and 2ADNT is less than a factor of three. This anomaly can be explained by the physical state of these analytes. RDX, TNT, and HMX are present as crystalline particulates, whereas 4ADNT and 2ADNT are formed only following dissolution and subsequent biotransformation. Therefore, one group of energetic materials exists as discrete particles while the other exists on the surfaces of soil grains and has had the opportunity to disperse into the surrounding substrate. Because of this phenomenon, individual cell discrete data are inadequate to represent areas as small as one square meter when the analytes remain in a crystalline particulate state. Thus, any type of site characterization based on discrete samples for RDX, TNT, and HMX would not be valid. 


\begin{tabular}{|c|c|c|c|c|c|}
\hline \multirow[b]{2}{*}{ Field \# } & \multicolumn{5}{|c|}{ Soil concentration $(\mathrm{mg} / \mathrm{kg})$} \\
\hline & HMX & RDX & TNT & 4ADNT & 2ADNT \\
\hline D14* & 60.2 & 557 & 164 & 3.30 & 3.61 \\
\hline D14-FD† & 23.5 & 167 & 3.57 & 2.43 & 2.94 \\
\hline $\mathrm{C} 14^{\star \star}$ & 18.5 & 139 & 15.5 & 2.24 & 2.88 \\
\hline D19 & 0.075 & 0.335 & 0.024 & 0.036 & 0.042 \\
\hline D19-FD & 0.067 & 0.225 & $<d$ & 0.046 & 0.061 \\
\hline C19 & 0.364 & 2.40 & 0.196 & 0.106 & 0.110 \\
\hline D27 & 0.528 & 3.75 & 0.022 & 0.153 & 0.182 \\
\hline D27-FD & 0.342 & 1.68 & 0.025 & 0.240 & 0.278 \\
\hline $\mathrm{C} 27$ & 3.24 & 24.2 & 6.10 & 0.562 & 0.604 \\
\hline D28 & 0.185 & 0.618 & 0.002 & 0.038 & 0.045 \\
\hline D28-FD & 0.195 & 0.693 & $<d$ & 0.058 & 0.080 \\
\hline $\mathrm{C} 28$ & 3.24 & 26.8 & 7.46 & 0.732 & 0.712 \\
\hline D29 & 0.077 & 0.193 & 0.004 & 0.034 & 0.040 \\
\hline D29-FD & 0.016 & 0.092 & $<d$ & 0.010 & 0.012 \\
\hline $\mathrm{C} 29$ & 0.258 & 1.24 & 0.084 & 0.050 & 0.070 \\
\hline D34 & 1.42 & 10.6 & 3.47 & 0.566 & 0.691 \\
\hline D34-FD & 24.3 & 203 & 45.9 & 1.49 & 1.49 \\
\hline C34 & 3.20 & 26.4 & 6.58 & 1.09 & 1.21 \\
\hline D39 & 0.067 & 0.175 & 0.009 & 0.049 & 0.053 \\
\hline D39-FD & 0.239 & 3.31 & 0.009 & 0.040 & 0.038 \\
\hline C39 & 0.063 & 0.522 & 0.009 & 0.039 & 0.044 \\
\hline D44 & 0.108 & 0.571 & 0.009 & 0.347 & 0.471 \\
\hline D44-FD & 0.137 & 0.540 & 0.031 & 0.708 & 0.950 \\
\hline C44 & 1.03 & 8.68 & 1.54 & 0.620 & 0.712 \\
\hline D46 & 2.52 & 19.9 & 3.94 & 0.711 & 0.668 \\
\hline D46-FD & 0.55 & 6.54 & 1.55 & 0.259 & 0.282 \\
\hline C46 & 0.29 & 1.85 & 0.16 & 0.098 & 0.124 \\
\hline
\end{tabular}




\begin{tabular}{|c|c|c|c|c|c|}
\hline \multicolumn{6}{|c|}{ Table 5 (cont'd). } \\
\hline \multirow[b]{2}{*}{ Field \# } & \multicolumn{5}{|c|}{ Soil concentration (mg/kg) } \\
\hline & HMX & RDX & TNT & 4ADNT & 2ADNT \\
\hline D49 & 0.092 & 1.46 & 0.004 & 0.015 & 0.018 \\
\hline D49-FD & 0.049 & 0.166 & 0.009 & $<d$ & 0.022 \\
\hline C49 & 0.062 & 0.266 & 0.008 & 0.026 & 0.036 \\
\hline D51 & 34.9 & 331 & 81.6 & 2.23 & 2.15 \\
\hline D51-FD & 5.87 & 40.9 & 2.01 & 1.08 & 1.10 \\
\hline C51 & 3.04 & 21.6 & 2.68 & 0.640 & 0.460 \\
\hline D55 & 0.517 & 3.67 & 1.31 & 0.108 & 0.104 \\
\hline D55-FD & 0.589 & 2.68 & 0.10 & 0.222 & 0.274 \\
\hline C55 & 0.934 & 8.18 & 2.52 & 0.196 & 0.198 \\
\hline D56 & 0.043 & 0.243 & $<d$ & 0.023 & 0.027 \\
\hline D56-FD & 0.299 & 2.40 & 0.350 & 0.074 & 0.089 \\
\hline C56 & 1.05 & 13.1 & 0.126 & 0.066 & 0.070 \\
\hline D72 & 0.468 & 1.40 & 0.054 & 0.755 & 0.813 \\
\hline D72-FD & 0.126 & 0.61 & 0.036 & 0.636 & 0.771 \\
\hline $\mathrm{C} 72$ & 0.968 & 6.88 & 1.19 & 1.07 & 1.08 \\
\hline D80 & 0.058 & 0.187 & 0.004 & 0.017 & 0.031 \\
\hline D80-FD & 17.2 & 179 & 118 & 0.081 & 0.065 \\
\hline $\mathrm{C} 80$ & 0.052 & 0.266 & 0.036 & 0.022 & 0.036 \\
\hline D82 & 2.80 & 24.1 & 3.29 & 1.11 & 1.09 \\
\hline D82-FD & 1.22 & 9.64 & 0.60 & 0.67 & 0.71 \\
\hline C82 & 0.69 & 4.50 & 0.47 & 0.33 & 0.33 \\
\hline D83 & 1.55 & 7.73 & 0.783 & 0.479 & 0.550 \\
\hline D83-FD & 0.76 & 3.72 & 0.213 & 0.248 & 0.255 \\
\hline $\mathrm{C} 83$ & 0.99 & 5.48 & 0.394 & 0.494 & 0.564 \\
\hline D87 & 0.076 & 0.366 & 0.003 & 0.021 & 0.049 \\
\hline D87-FD & 0.071 & 0.255 & 0.004 & 0.071 & 0.089 \\
\hline $\mathrm{C} 87$ & 0.228 & 1.504 & 37.4 & 0.278 & 0.260 \\
\hline D90 & 0.108 & 0.138 & 0.004 & 0.022 & 0.022 \\
\hline D90-FD & 0.035 & 0.058 & $<\mathrm{d}$ & 0.013 & 0.016 \\
\hline $\mathrm{C} 90$ & 0.056 & 0.168 & 0.190 & 0.022 & 0.024 \\
\hline
\end{tabular}




\begin{tabular}{|c|c|c|c|c|c|}
\hline \multirow[b]{2}{*}{ Field \# } & \multicolumn{5}{|c|}{ Soil concentration $(\mathrm{mg} / \mathrm{kg})$} \\
\hline & HMX & RDX & TNT & 4ADNT & 2ADNT \\
\hline D100 & $<d$ & 0.067 & $<d$ & 0.013 & 0.022 \\
\hline D100-FD & 1.15 & 18.8 & 1.18 & 0.051 & 0.063 \\
\hline C100 & 0.282 & 2.92 & 0.982 & 0.068 & 0.070 \\
\hline \multicolumn{6}{|c|}{ * $\quad$ Minigrid discrete samples. } \\
\hline \multicolumn{6}{|c|}{ ** Ten-increment minigrid composite samples. } \\
\hline
\end{tabular}

Results for the ten-increment composite samples collected within the same randomly selected minigrids where duplicate discrete samples were collected are also presented in Table 5. These results exhibit the same trends as the field duplicate discrete samples. However, since the composite samples comprise about 5\% of the surface area of the 1 - $\mathrm{m} \times 1$-m minigrid, they should provide a better estimate of the analyte concentration than the discrete samples that comprise only $0.5 \%$ of the surface area of the minigrid.

\section{Results for 25-increment composite samples collected within the 10-m × 10-m grid near low-order detonation}

Analytical results for the ten random 25-increment composite samples collected within the entire $10-\mathrm{m} \times 10$-m grid are shown in Table 6 . The minimum and maximum concentrations for RDX were 4.62 and $294 \mathrm{mg} / \mathrm{kg}$, respectively. This range is only a factor of about 64 , whereas the range of concentrations found for the 100 discrete samples from this area differed by nearly five orders of magnitude. However, the relative standard deviation for RDX in these composites was $159 \%$ and the median and mean differed by a factor of 2.2, indicating that this group of data for 25-increment composites was not normally distributed. Clearly, very different values can result for random composites, depending on whether or not increments were collected from the apparent hot spots shown in Figure 9. Moreover, because of the presence of a hot spot, energetic residue distribution is clearly not uniform in this grid. 
Table 6. Results from the analysis of 25-increment composite samples from grid near a low-order 81-mm mortar detonation at Fort Polk.

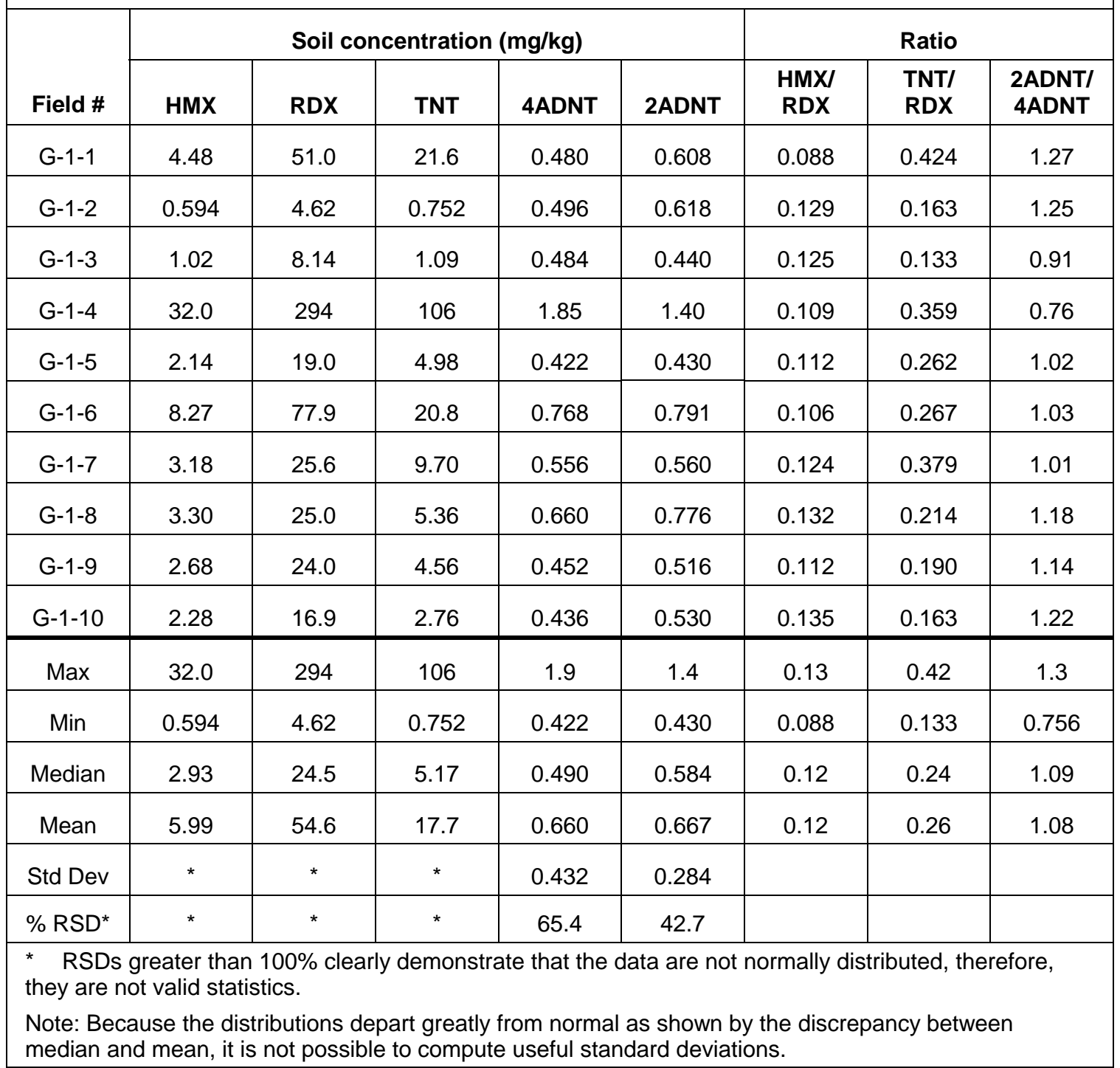

Recent results from sampling at Canadian Force Base-Gagetown (Thiboutot et al. in press) found that multi-increment composite samples collected systematically within a specified area provided reproducible results. We evaluated this approach for this study by creating four mathematical systematic composite samples $(n=25)$ by combining every fourth discrete sample from the 100 discrete minigrids. A comparison of the results for the 100 discrete samples, the ten randomly collected 25-increment composites, and the four systematic mathe- 
matical 25-increment composites is shown in Table 7. The range of RDX values is much reduced from a factor of about $10^{5}$ for the discrete samples to factors of 64 and 3, respectively, for the randomly and systematically collected composites. The results for the systematic samples come from only four samples and additional research is needed to verify that this sampling strategy generally provides more reproducible results for this set of environmental conditions. Moreover, it is recognized that the success of a systematic sampling strategy to be reproducible is dependent on the size of the hot spot and the spacing of the sample increments. Likewise, the reproducibility of a random sampling strategy depends on the number of increments. A critical variable for both sampling strategies is the dimensions of the hot spot, which in this case were most likely associated with the partial detonation of an 81-mm mortar.

\begin{tabular}{|c|c|c|c|c|c|}
\hline \multirow[b]{2}{*}{ Collection strategy } & \multirow{2}{*}{$\begin{array}{l}\text { Increments } \\
\text { per Sample }\end{array}$} & \multirow{2}{*}{$\begin{array}{l}\text { Number of } \\
\text { Replicates }\end{array}$} & \multicolumn{3}{|c|}{ Analytes } \\
\hline & & & RDX & HMX & TNT \\
\hline Discrete samples & 1 & 100 & & & \\
\hline $\operatorname{Max}$ & & & 2390 & 253 & 1560 \\
\hline Min & & & 0.037 & 0.005 & 0.001 \\
\hline Mean & & & 70.9 & 7.89 & 29.7 \\
\hline Median & & & 1.79 & 0.395 & 0.044 \\
\hline Composite (random) & 25 & 10 & & & \\
\hline Max & & & 294 & 32 & 106 \\
\hline Min & & & 4.62 & 0.594 & 0.752 \\
\hline Mean & & & 54.6 & 5.99 & 17.7 \\
\hline Median & & & 24.5 & 2.93 & 5.17 \\
\hline Composite (systematic) & 25 & 4 & & & \\
\hline Max & & & 99.8 & 10.8 & 63.2 \\
\hline Min & & & 33.1 & 3.81 & 10.3 \\
\hline Mean & & & $70.9^{\star}$ & $7.89 *$ & $29.7^{*}$ \\
\hline$\% \mathrm{RSD}$ & & & $43.3 \%$ & $40.0 \%$ & $77.8 \%$ \\
\hline Median & & & 75.3 & 8.46 & 22.7 \\
\hline
\end{tabular}

It is also interesting to compare the median value obtained from the 100 discrete samples with that from the ten 25-increment random and four 25increment systematic composite samples, because these median values represent 
the concentration that half of the sample collected will have lower than (or higher than) values, for this grid. The median of the discrete samples for RDX was 1.79 $\mathrm{mg} / \mathrm{kg}$, the median for the ten composite samples was $24.5 \mathrm{mg} / \mathrm{kg}$, and the median for the four systematic samples was $75.3 \mathrm{mg} / \mathrm{kg}$. Thus, rather than diluting out the high concentrations, the multi-increment composite samples are more likely to capture the high concentrations that the discrete samples often miss. A comparison of the discrete and composite (random and systematic) medians for HMX and TNT results in a very similar trend, much higher median concentrations for the composite samples: 0.395 versus 2.93 and $8.5 \mathrm{mg} / \mathrm{kg}$ for HMX and 0.044 versus 5.17 and $22.7 \mathrm{mg} / \mathrm{kg}$ for TNT, respectively (Table 7).

\begin{tabular}{|c|}
\hline Grid size $=10 \mathrm{~m} \times 10 \mathrm{~m}$ \\
\hline Grid area $=100 \mathrm{~m}^{2}$ \\
\hline Sample depth $=0.025 \mathrm{~m}$ \\
\hline Volume of soil sampled $=2.5 \mathrm{~m}^{3}=2.5 \mathrm{E} 6 \mathrm{~cm}^{3}$ \\
\hline Soil density $=1.7 \mathrm{~g} / \mathrm{cm}^{3}$ \\
\hline Mass of soil $=1.7 \mathrm{~g} / \mathrm{cm}^{3} \times 2.5 \mathrm{e} 6 \mathrm{~cm}^{3}=4.3 \mathrm{e} 6 \mathrm{~g}=4.3 \mathrm{e} 3 \mathrm{~kg}$ \\
\hline $\begin{array}{l}\text { Weighted average of the average (by total number of increments) RDX concentration for } \\
\text { the ten } 25 \text {-increment random composite samples and the } 100 \text { discrete samples } 0.059 \mathrm{~g} / \mathrm{kg}\end{array}$ \\
\hline Mass of RDX present in soil to $2.5-\mathrm{cm}$ depth: $0.0059 \mathrm{~g} / \mathrm{kg} \times 4.3 \mathrm{E} 3 \mathrm{~kg}=250 \mathrm{~g}$ \\
\hline Mass of Composition B collected from grid $=198 \mathrm{~g}$ \\
\hline Composition B is $54 \% \mathrm{RDX}, 6 \% \mathrm{HMX}, 39 \% \mathrm{TNT}, 1 \%$ wax. \\
\hline Mass of RDX in Composition B $=54 \%$ of $198 \mathrm{~g}=107 \mathrm{~g}$ \\
\hline
\end{tabular}

A calculation using the weighted average of the average RDX concentration for the ten 25-increment random composite samples and the 100 discrete was made to determine the total mass of RDX present in the topsoil of the $10-\mathrm{m} \times 10$ m grid surrounding the partial detonation event (Table 8). An additional calculation was then made that included both the surface-soil-associated RDX to a depth of $2.5 \mathrm{~cm}$, and the RDX associated with the chunks of Composition B found lying on the surface. The total mass was estimated at $360 \mathrm{~g} / 100 \mathrm{~m}^{2}$. By comparison, the $200-\mathrm{m}^{2}$ area sampled around the tank target had a median RDX concen- 
tration of $0.948 \mathrm{mg} / \mathrm{kg}$, resulting in a total mass of RDX of $2.04 \mathrm{~g} / 100 \mathrm{~m}^{2}$, which is two orders of magnitude lower than the mass in the area surrounding the 81mm mortar low-order detonation. Even though the target value is smaller than the partial detonation event area, it is three orders of magnitude more than values encountered in previous studies around targets on other artillery impact ranges (Pennington et al. 2001, 2002, 2003). Therefore, although no evidence such as "chunk" energetic residue or large munition fragments was observed, partial detonation events may have occurred near this tank target. In addition, if the partial detonation of an 81-mm mortar was the only source of this energetic residue, then more than one-half of the main charge (560 g RDX) was present on or in the near surface of the $10-\mathrm{m} \times 10$-m grid. Indeed, most of the mass exists as particles that would fit into the size category used for soil (i.e., $<2 \mathrm{~mm}$, Table 8).

\section{Line composite samples surrounding 10-m × 10-m grid}

Analytical results for the line composite samples collected at 2-m, 5-m, and $10-\mathrm{m}$ distances off the four edges of the $10-\mathrm{m} \times 10-\mathrm{m}$ grid are presented in Table 9 and Figure 11. The concentrations of RDX at $2 \mathrm{~m}$ off the south and east edges of the grid are 11.3 and $4.56 \mathrm{mg} / \mathrm{kg}$, respectively, which is consistent with the locations where high soil concentrations and particles of solid Composition B were found (Figures 9, 10). The ratios of HMX/RDX and TNT/RDX for the line composite samples are quite similar to those for the discrete minigrid samples, indicating that the contamination is from the same source (i.e., same extent of weathering) Composition B.

For the south, east, and west sides of the grid, concentrations of all analytes decrease as distance from the edge of the grid increases. For the north samples, the highest values for HMX and RDX are found in the 5-m sample. In all cases the samples at a distance of $10 \mathrm{~m}$ from the edge are very low compared with most of the concentrations within the grid and the samples collected at 2 and $5 \mathrm{~m}$ from the edge. Thus it appears that we have captured the area impacted to the greatest extent from the low-order detonation within the 30-m $\times 30$-m area sampled.

\section{Physical size of hot spot from low-order 81-mm mortar round}

In order to develop strategies for hot spot detection, typical physical sizes of these hot spots must be known. It is anticipated that the dimensions of hot spots will depend on both the type of munition and the failure mechanism. Within this $10-\mathrm{m} \times 10-\mathrm{m}$ area, the major hot spot appears to be centered at minigrid D15. However, since this is based on discrete samples, caution must be stressed, and the subsequent size estimate is tentative. Inspection of Figures 9 and 10 indicates that if we use a concentration of $100 \mathrm{mg} / \mathrm{kg}$ as an indicator of the hot spot, the 
size of the hot spot around minigrid D15 is about $3 \mathrm{~m} \times 3 \mathrm{~m}$. This is only the first estimate of this type and additional research is planned to provide additional data for dimensions of hot spots from partial detonations of various ordnance items.

\begin{tabular}{|c|c|c|c|c|c|c|c|c|}
\hline \multirow[b]{2}{*}{$\begin{array}{c}\text { Edge } \\
\text { samples }\end{array}$} & \multicolumn{6}{|c|}{ Soil concentration (mg/kg) } & \multicolumn{2}{|c|}{ Ratio } \\
\hline & HMX & RDX & TNT & 2,4-DNT & 4ADNT & 2ADNT & $\begin{array}{l}\text { HMXI } \\
\text { RDX }\end{array}$ & $\begin{array}{l}\text { TNTI } \\
\text { RDX }\end{array}$ \\
\hline $2 \mathrm{~m}$ south & 1.39 & 11.3 & 2.56 & $<d$ & 0.444 & 0.462 & 0.123 & 0.227 \\
\hline $5 \mathrm{~m}$ south & 0.316 & 1.82 & 0.352 & $<\mathrm{d}$ & 0.144 & 0.192 & 0.173 & 0.193 \\
\hline 10 m south & 0.076 & 0.212 & 0.308 & $<d$ & 0.130 & 0.190 & 0.358 & 1.453 \\
\hline $2 \mathrm{~m}$ east & 0.614 & 4.56 & 1.20 & $<\mathrm{d}$ & 0.149 & 0.156 & 0.135 & 0.262 \\
\hline $5 \mathrm{~m}$ east & 0.180 & 1.19 & 0.288 & $<\mathrm{d}$ & 0.070 & 0.060 & 0.151 & 0.242 \\
\hline 10 m east & 0.098 & 0.508 & 0.040 & $<\mathrm{d}$ & 0.030 & 0.050 & 0.193 & 0.079 \\
\hline $2 \mathrm{~m}$ north & $<d$ & 0.130 & 0.014 & 0.004 & 0.020 & 0.024 & & 0.108 \\
\hline $5 \mathrm{~m}$ north & 0.130 & 2.25 & $<d$ & 0.004 & 0.014 & 0.016 & 0.058 & \\
\hline 10 m north & 0.062 & 0.198 & 0.002 & $<d$ & 0.006 & 0.008 & 0.313 & 0.010 \\
\hline $2 \mathrm{~m}$ west & 0.226 & 0.930 & 0.038 & 0.006 & 0.072 & 0.078 & 0.243 & 0.041 \\
\hline $5 \mathrm{~m}$ west & 0.134 & 0.618 & 0.028 & 0.004 & 0.066 & 0.066 & 0.217 & 0.045 \\
\hline \multirow[t]{3}{*}{$10 \mathrm{~m}$ west } & 0.068 & 0.288 & 0.020 & 0.004 & 0.044 & 0.046 & 0.236 & 0.069 \\
\hline & & & & & & Mean & 0.200 & 0.248 \\
\hline & & & & & & Median & 0.193 & 0.108 \\
\hline
\end{tabular}




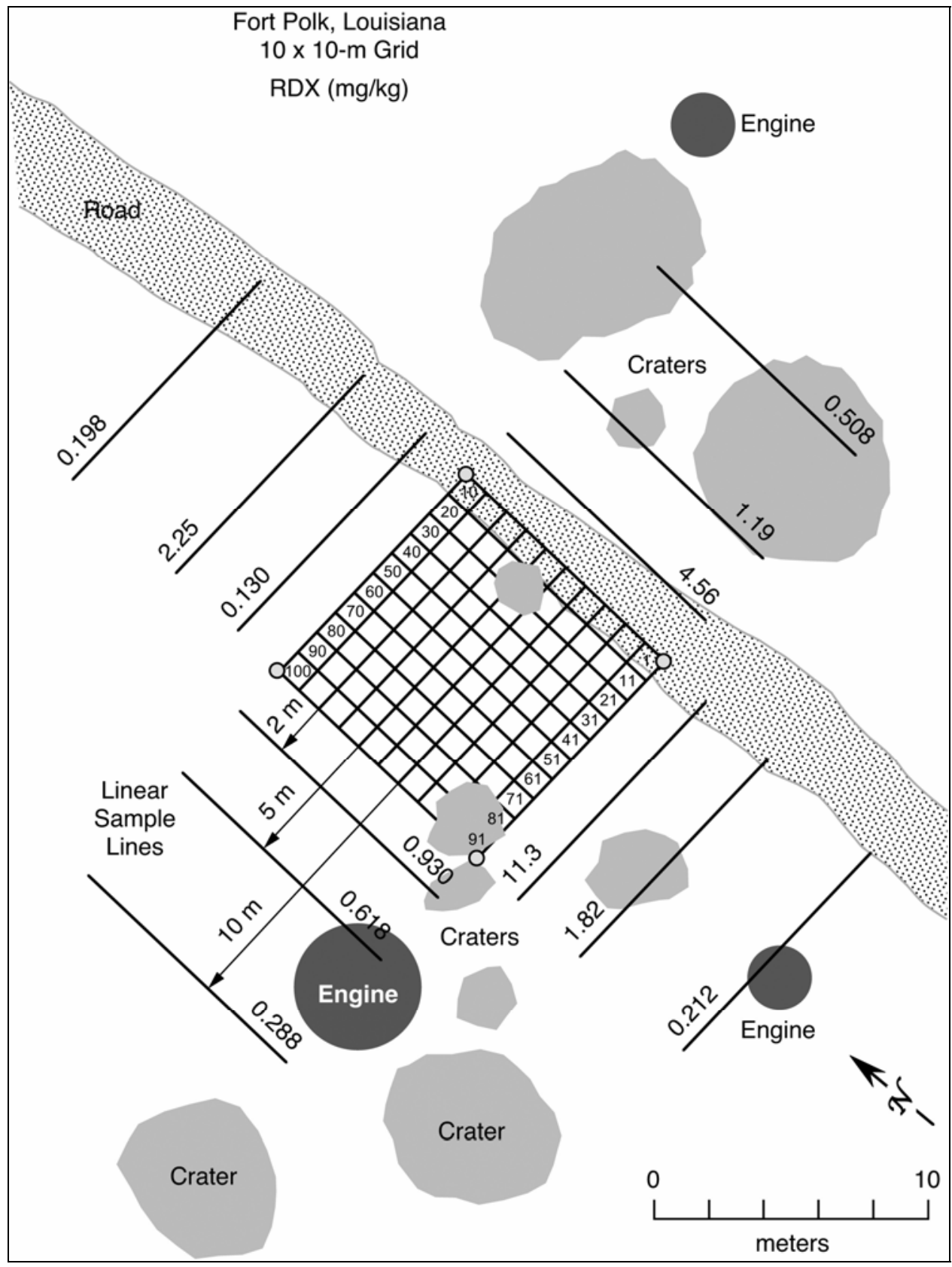

Figure 11. Soil RDX concentrations in linear composite samples taken at various distances from the sampling grid. 


\section{Target analyte concentrations near an artillery target}

Target analyte concentrations for ten-increment composite surface soil samples that we collected near an artillery target are presented in Table 10 and Figure 12. The concentrations of RDX varied from 0.106 to $15.9 \mathrm{mg} / \mathrm{kg}$, but unlike HMX concentrations near an antitank target (Jenkins et al. 1997, 1998, 2004), there does not appear to be a concentration gradient relative to distance from the target. Therefore, there appears to be a difference in residue pattern around a line-of-sight target as opposed to a target that receives mostly indirect fire. TNT concentrations in these samples varied from 0.076 to $18.8 \mathrm{mg} / \mathrm{kg}$, and the ratios of TNT to RDX were often higher than the 0.7 ratio expected from deposition of fresh Composition B (Table 10). Most 155-mm artillery rounds are filled with TNT rather than Composition B and it appears from the ratio of TNT/ RDX that a portion of the explosives residues detected near this target was from TNT-filled rounds. The ratio of HMX to RDX in these samples was also often higher than found in and near the 10-m $\times 10$-m grid located downhill and to the right of this target. This implies that the Composition $\mathrm{B}$ residues near this target are somewhat older (i.e., more weathered) than those near the low-order 81-mm mortar round. This phenomenon occurs because RDX will dissolve faster and migrate away from these residues faster than HMX as a result of its higher aqueous solubility, which exceeds HMX by about a factor of 10 .

Table 10. Target analyte concentrations in area around an artillery target in the impact area, Fort Polk.

\begin{tabular}{|c|c|c|c|c|c|c|c|c|c|}
\hline & \multicolumn{6}{|c|}{ Soil concentration (mg/kg) } & \multicolumn{3}{|c|}{ Ratio } \\
\hline & HMX & TNB & RDX & TNT & 4ADNT & 2ADNT & $\begin{array}{l}\text { HMXI } \\
\text { RDX }\end{array}$ & $\begin{array}{l}\text { TNTI } \\
\text { RDX }\end{array}$ & $\begin{array}{l}\text { 2ADNTI } \\
\text { 4ADNT }\end{array}$ \\
\hline NE 0-2 m & 15.2 & $<d$ & 15.9 & 1.19 & 0.246 & 0.308 & 0.956 & 0.075 & 1.25 \\
\hline NW 0-2 m & 1.43 & $<d$ & 1.17 & 0.144 & 0.170 & 0.206 & 1.22 & 0.123 & 1.21 \\
\hline SW 0-2 m & 0.420 & $<d$ & 2.18 & 0.516 & 0.280 & 0.356 & 0.193 & 0.237 & 1.27 \\
\hline SE 0-2 m & 0.360 & 0.082 & 0.500 & 18.8 & 0.912 & 1.17 & 0.720 & 37.7 & 1.28 \\
\hline ENE 2-5 m & 0.876 & $<d$ & 0.448 & 0.436 & 0.172 & 0.230 & 1.96 & 0.973 & 1.34 \\
\hline NNE 2-5 m & 0.236 & $<d$ & 0.724 & 0.076 & 0.074 & 0.096 & 0.326 & 0.105 & 1.30 \\
\hline NNW 2-5 m & 0.216 & $<d$ & 1.75 & 14.5 & 0.266 & 0.248 & 0.123 & 8.24 & 0.93 \\
\hline WNW 2-5 m & 0.120 & $<d$ & 0.422 & 0.234 & 0.176 & 0.274 & 0.284 & 0.555 & 1.56 \\
\hline WSW $2-5 \mathrm{~m}$ & 1.92 & $<d$ & 13.3 & 4.42 & 0.526 & 0.732 & 0.144 & 0.333 & 1.39 \\
\hline SSW 2-5 m & 0.225 & $<d$ & 1.23 & 2.22 & 0.611 & 0.875 & 0.183 & 1.81 & 1.43 \\
\hline SSE 2-5 m & 0.134 & $<d$ & 0.294 & 9.46 & 1.14 & 1.42 & 0.456 & 32.2 & 1.25 \\
\hline \multirow[t]{3}{*}{ ESE 2-5 m } & 0.064 & $<d$ & 0.106 & 0.782 & 0.296 & 0.400 & 0.604 & 7.38 & 1.35 \\
\hline & & & & & & Mean & 0.597 & 7.47 & 1.30 \\
\hline & & & & & & Median & 0.391 & 0.764 & 1.29 \\
\hline
\end{tabular}




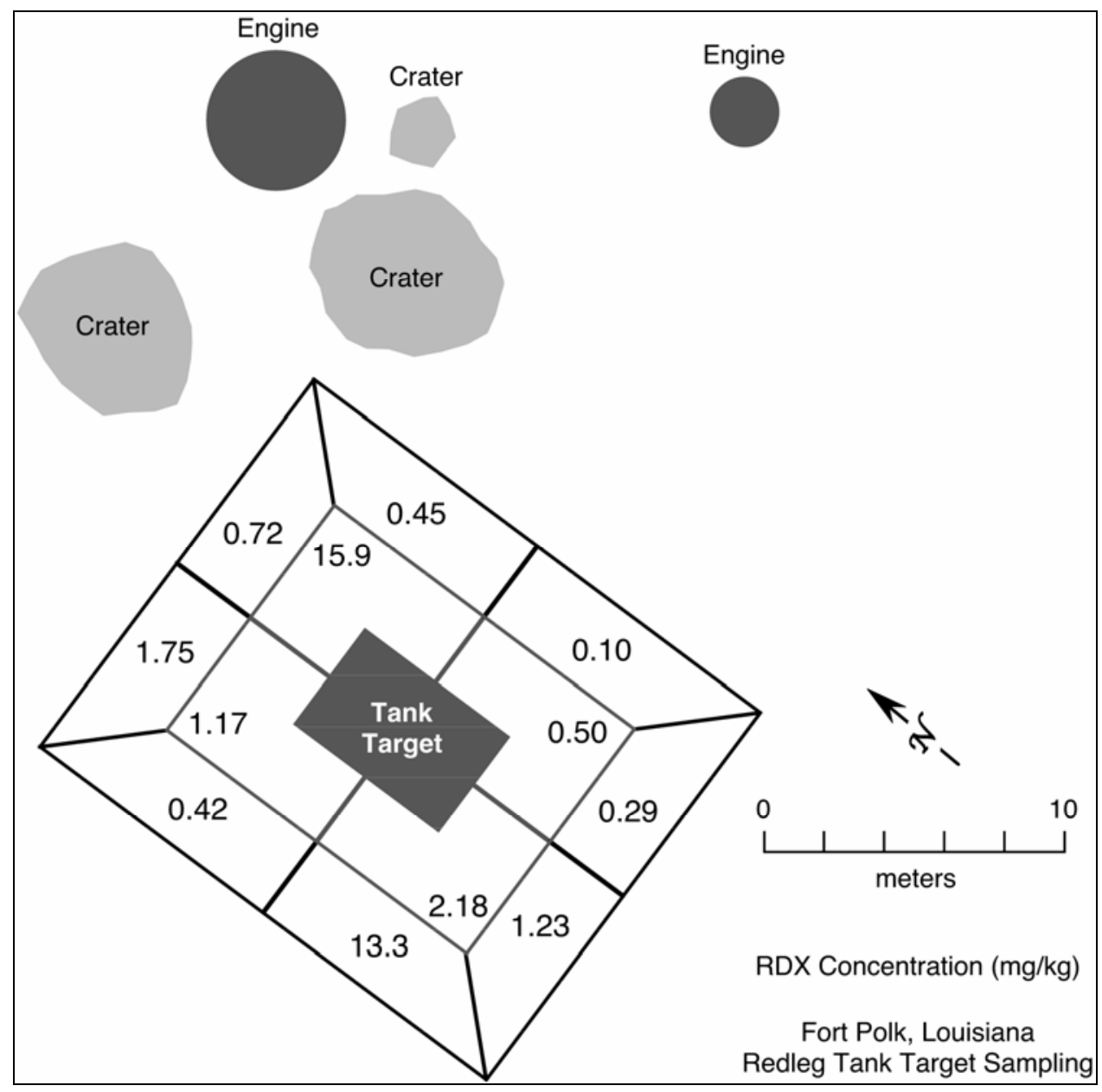

Figure 12. Soil concentration of RDX taken in various sampling areas around a tank target. 


\section{SUMMARY AND CONCLUSIONS}

Surface soil samples were collected and analyzed for explosives residues from the artillery/mortar impact area located at Fort Polk, Louisiana. Two distinct areas were selected for sampling. The first was around a low-order detonation event and the second around a tank target.

The first sampling area was selected as a result of the observation of numerous pieces of Composition $\mathrm{B}$ residue lying on the soil surface, thereby giving us the opportunity to sample a potential "hot spot." A 10-m $\times 10$-m sampling grid that encompassed the residue chunks of Composition B was laid out. This grid was further subdivided into 1001 -m $\times 1$-m minigrids. Observed pieces of Composition $\mathrm{B}$ were collected and weighed and their position within the grid system was cataloged. One discrete soil sample was collected from each of the minigrids. The most predominant analyte, RDX, had concentrations ranging over approximately five orders of magnitude for the 100 discrete samples. TNT concentrations ranged from less than detect $(0.002 \mathrm{mg} / \mathrm{kg})$ to $1560 \mathrm{mg} / \mathrm{kg}$ or six orders of magnitude. Certainly no single discrete sample could accurately represent the entire grid area.

Field duplicate discrete samples were collected from 20 randomly chosen minigrids. The differences between these field duplicates varied up to three orders of magnitude, indicating that single samples cannot represent areas as small as one minigrid (square meter) for energetic materials that exist as crystalline particulates. Ten-increment composites were also collected within these twenty minigrids. There was no apparent agreement between the initial discrete and the composite samples, indicating again that the discrete sampling method does not represent the area of concern for RDX, TNT, and HMX. Ten composite samples of 25 randomly chosen increments each were taken over the entire 10-m $\times 10-\mathrm{m}$ grid. The median RDX concentration for the ten composite samples was fourteen times higher than the median of the 100 discrete samples because the "hot spots" were more frequently sampled with the former sampling strategy than were the latter. Also, RDX concentrations for these composite samples varied as much as 60 times as a result of the number of times the "hot spot” was sampled. Therefore, under these conditions, much uncertainty exists among composite samples composed of 25 randomly collected increments, even though a large improvement over discrete sampling was achieved.

We decided to mathematically generate systematic random samples by "compositing," from the 100 discrete samples, every fourth minigrid. We did this four times, thereby using all of the minigrid samples. The median value for RDX was 
three times greater than the ten random composites median and 42 times the median for the discrete samples. The RDX concentration range for the results of these four systematic mathematical composites was 33 to $100 \mathrm{mg} / \mathrm{kg}$, whereas the range was 5 to $294 \mathrm{mg} / \mathrm{kg}$, and 0.04 to $2,390 \mathrm{mg} / \mathrm{kg}$ for the ten 25 -increment randomly collected composites and the 100 discrete samples, respectively. It appears the systematic approach is more likely to consistently sample "hot spots" of the size encountered in this study.

Because most of the discrete samples had concentrations well below the mean, we divided the results into those less or greater than $100 \mathrm{mg} / \mathrm{kg}$ in order to delineate the area of highest concentration. The majority of these later samples were co-located within an area of approximately $3 \mathrm{~m} \times 3 \mathrm{~m}$. The remaining high values were from two diagonally joined minigrids.

Composite soil samples were also collected along linear transects at 2, 5, and $10 \mathrm{~m}$ from the grid on all four sides. RDX concentrations decreased with distance from the grid to less than $0.3 \mathrm{mg} / \mathrm{kg}$ at the 10 -m distances. It appears that this detonation event extended its influence across an area of approximately $30 \mathrm{~m} \times$ $30 \mathrm{~m}$ if one chose a boundary concentration of $0.3 \mathrm{mg} / \mathrm{kg}$.

The second sampling area, a tank target, was selected because of the fact that it would be a point of interest for incoming fire. A sampling scheme different from that used to delineate the area of influence of the low-order detonation event was used at the target. A two-meter-from-the-target grid was set around the tank and then quartered. An additional grid was set at $5 \mathrm{~m}$ from the tank and this 2- to 5-m area was divided into eight parts. Ten-increment composite soil samples were collected from inside each of the twelve areas. RDX concentrations varied from 0.1 to $16 \mathrm{mg} / \mathrm{kg}$. Within the described target sampling area there was no apparent pattern to the distribution of RDX, i.e., there was no concentration gradient moving out from the target, such as those found around targets at antitank ranges. One might anticipate this, as direct fire from firing point to target is used at antitank ranges. This means that the munition comes from one direction and usually impacts the target. Any casing rupture or partial detonation would be at the target and the majority of HE would be deposited there and spattering would decrease with distance from the target. At artillery and mortar impact areas, the HE round can arrive from the air from numerous directions, frequently from all around the target. Partial detonation events could occur anywhere around the target and possibly at considerable distance from the target, depending on the accuracy of the gunner.

This study reinforces earlier work, the results of which indicated that loworder (partial) detonation events produce the most HE residues within impact areas. In most cases pure HE material is found and residue concentrations in the 
soil are higher than the surrounding area. This work was a first attempt to delineate the area of influence of one of these events. Similar studies need to be made to provide more estimates of the areas influenced by the low-order detonations of other munitions. Additionally, systematic random composites seem to produce a more reproducible and regulatory appropriate sample than that generated by a random composite, and certainly either provides results that are an improvement over discrete sampling methods. Additional field studies are needed to compare systematic random composites with random composites under a variety of conditions and events. Also, sampling studies need to be conducted over larger areas than the $10-\mathrm{m} \times 10$ - $\mathrm{m}$ area studied here. 


\section{REFERENCES}

Ampleman, G., S. Thiboutot, J. Lewis, A. Marois, A. Gagnon, M. Bouchard, R. Martel, R. Lefebvre, T.A. Ranney, T.F. Jenkins, and J.C. Pennington (2003a) Evaluation of the impacts of live-fire training at CFB Shilo (final report). Defence Research Development Canada-Valcartier, Technical Report TR 2003066, April 2003.

Ampleman, G., S. Thiboutot, J. Lewis, A. Marois, A. Gagnon, M. Bouchard, S. Jean, T. Jenkins, A. Hewitt, J.C. Pennington, and T.A. Ranney (2003b) Evaluation of the contamination by explosives at Cold Lake Air Weapons Range (CLAWR), Alberta: Phase 1 Report. Defence Research Development CanadaValcartier, Technical Report TR 2003-208, December 2003.

Environmental Protection Agency (1994) Nitroaromatics and nitramines by HPLC. Second Update SW846 Method 8330.

Environmental Protection Agency (1999) Nitroaromatics and nitramines by GC-ECD. Fourth Update SW846 Method 8095.

Hewitt, A.D., and M.E. Walsh (2003) On-site processing and subsampling of surface soils samples for the analysis of explosives. U.S. Army Engineer Research and Development Center, Hanover, New Hampshire, Technical Report ERDC/CRREL TR-03-14.

Jenkins, T.F., C.L. Grant, G.S. Brar, P.G. Thorne, T.A. Ranney, and P.W. Schumacher (1996) Assessment of sampling error associated with collection and analysis of soil samples at explosives-contaminated sites. U.S. Army Cold Regions Research and Engineering Laboratory, Hanover, New Hampshire, Special Report 96-15.

Jenkins, T.F., M.E. Walsh, P.G. Thorne, S. Thiboutot, G. Ampleman, T.A. Ranney, and C.L. Grant (1997) Assessment of sampling error associated with the collection and analysis of soil samples at a firing range contaminated with HMX. U.S. Army Cold Regions Research and Engineering Laboratory, Hanover, New Hampshire, Special Report 97-22.

Jenkins, T.F., M.E. Walsh, P.G. Thorne, P.H. Miyares, T.A. Ranney, C.L. Grant, and J. Esparza (1998) Site characterization for explosives at a military firing range impact area. U.S. Army Cold Regions Research and Engineering Laboratory, Hanover, New Hampshire, Special Report 98-9. 
Jenkins, T.F., C.L. Grant, M.E. Walsh, P.G. Thorne, S. Thiboutot, G. Ampleman, and T.A. Ranney (1999) Coping with spatial heterogeneity effects on sampling and analysis at an HMX-contaminated antitank firing range. Field Analytical Chemistry and Technology, 3(1): 19-28.

Jenkins, T.F., J.C. Pennington, T.A. Ranney, T.E. Berry, P.H. Miyares, M.E. Walsh, A.D. Hewitt, N.M. Perron, L.V. Parker, C.A. Hayes, and E. Wahlgren (2001) Characterization of explosives contamination at military firing ranges. U.S. Army Engineer Research and Development Center, Cold Regions Research and Engineering Laboratory, Hanover, New Hampshire, Technical Report TR-01-5.

Jenkins, T.F., T.A. Ranney, A.D. Hewitt, M.E. Walsh, and K.L. Bjella (2004) Representative sampling for energetic compounds at an antitank firing range. U.S. Army Engineer Research and Development Center, Cold Regions Research and Engineering Laboratory, Hanover, New Hampshire, Technical Report TR04-7.

Pennington, J.C., T.F. Jenkins, P.H. Miyares, M.E. Walsh, A.D. Hewitt, N. Perron, T.A. Ranney, J. Lynch, J.J. Delfino, and C.A. Hayes (2001) Distribution and fate of energetics on DoD test and training ranges: Interim Report 1. U.S. Army Engineer Research and Development Center, Environmental Laboratory, Vicksburg, Mississippi, ERDC Technical Report TR-01-13.

Pennington, J.C., T.F. Jenkins, G. Ampleman, S. Thiboutot, J.M. Brannon, J. Lynch, T.A. Ranney, J. Stark, M.E. Walsh, J. Lewis, C.A. Hayes, J.E. Mirecki, A.D. Hewitt, N. Perron, D. Lambert, J. Clausen, and J.J. Delfino (2002) Distribution and fate of energetics on DoD test and training ranges: Interim Report 2. U.S. Army Engineer Research and Development Center, Environmental Laboratory, Vicksburg, Mississippi, ERDC Technical Report TR-028.

Pennington, J.C., T.F. Jenkins, G. Ampleman, S. Thiboutot, J.M. Brannon, J. Lewis, J.E. DeLaney, J. Clausen, A.D. Hewitt, M.A. Hollander, C.A. Hayes, J.A. Stark, A. Marois, S. Brochu, H.Q. Dinh, D. Lambert, A. Gagnon, M. Bouchard, R. Martel, P. Brousseau, N.M. Perron, R. Lefebvre, W. Davis, T.A. Ranney, C. Gauthier, S. Taylor, and J. Ballard (2003) Distribution and fate of energetics on DoD test and training ranges: Interim Report 3. U.S. Army Engineer Research and Development Center, Environmental Laboratory, Vicksburg, Mississippi, ERDC Technical Report TR-03-2. 
Thiboutot, S., G. Ampleman, A. Gagnon, A. Marois, T.F. Jenkins, M.E. Walsh, P.G. Thorne, and T.A. Ranney (1998) Characterization of antitank firing ranges at CFB Valcartier, WATC Wainwright, and CFAD Dundurn. Defence Research Establishment-Valcartier, Quebec, Report \# DREV-R-9809.

Thiboutot, S., G. Ampleman, J. Lewis, D. Faucher, A. Marois, R. Martel, J.M. Ballard, S. Downe, T. Jenkins, and A. Hewitt (2003) Environmental conditions of surface soils and biomass prevailing in the training area at CFB Gagetown, New Brunswick. Defence Research and Development CanadaValcartier, Quebec, Technical Report TR-2003-152.

Thiboutot, S., G. Ampleman, A. Marois, A. Gagnon, M. Bouchard, A. Hewitt, T. Jenkins, M. Walsh, and K. Bjella (in press) Environmental condition of surface soils, CFB Gagetown Training Area: Delineation of the presence of munitions-related residues (Phase III, Final Report). Defence Research and Development Canada Valcartier, DRDC Valcartier technical report.

Walsh, M.E., C.M. Collins, C.H. Racine, T.F. Jenkins, A.B. Gelvin, and T.A. Ranney (2001) Sampling for explosives residues at Fort Greely, Alaska: Reconnaissance visit July 2000. U.S. Army Engineer Research and Development Center, Hanover, New Hampshire, Technical Report ERDC/CRREL TR-01-15.

Walsh, M.E., C.M. Collins, A.D. Hewitt, M.R. Walsh, T.F Jenkins, J. Stark, A. Gelvin, T. Douglas, N. Perron, D. Lambert, R. Bailey, and K. Myers (2004) Range characterization studies at Donnelly Training Area, Alaska 2001 and 2002. U.S. Army Engineer Research and Development Center, Hanover, New Hampshire, Technical Report ERDC/CRREL TR-04-3.

Walsh, M.R. (2004) Field sampling tools for explosives residues developed at CRREL. U.S. Army Engineer Research and Development Center, Hanover, New Hampshire, Technical Note ERDC/CRREL TN-04-1. 


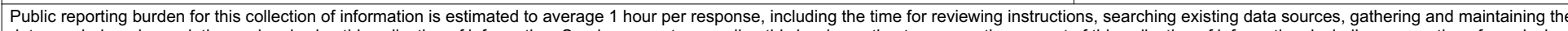

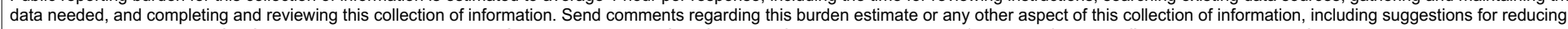

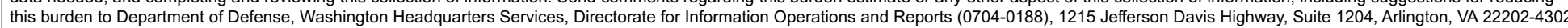

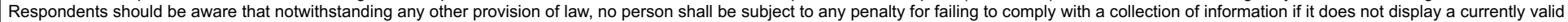
OMB control number. PLEASE DO NOT RETURN YOUR FORM TO THE ABOVE ADDRESS.
1. REPORT DATE (DD-MM-YY)
2. REPORT TYPE
DATES COVERED (From - To)

November 2004

Technical Report

4. TITLE AND SUBTITLE

Sampling Strategies Near a Low-Order Detonation and a

Target at an Artillery Impact Area

5a. CONTRACT NUMBER

5b. GRANT NUMBER

5c. PROGRAM ELEMENT NUMBER

\section{AUTHOR(S)}

5d. PROJECT NUMBER

Thomas F. Jenkins, Alan D. Hewitt, Thomas A. Ranney, Charles A. Ramsey,

Dennis J. Lambert, Kevin L. Bjella, and Nancy M. Perron

5e. TASK NUMBER

5f. WORK UNIT NUMBER

8. PERFORMING ORGANIZATION REPORT

7. PERFORMING ORGANIZATION NAME(S) AND ADDRESS(ES)

U.S. Army Engineer Research and Development Center

Cold Regions Research and Engineering Laboratory

72 Lyme Road

ERDC/CRREL TR-04-14

Hanover, NH 03755-1290

9. SPONSORING/MONITORING AGENCY NAME(S) AND ADDRESS(ES)

10. SPONSOR / MONITOR'S ACRONYM(S)

11. SPONSOR / MONITOR'S REPORT

NUMBER(S)

\section{DISTRIBUTION / AVAILABILITY STATEMENT}

Approved for public release; distribution is unlimited.

Available from NTIS, Springfield, Virginia 22161.

13. SUPPLEMENTARY NOTES

\section{ABSTRACT}

Field sampling experiments were conducted at the firing range at Fort Polk, Louisiana. The objectives were to determine the spatial distribution and best approach for collecting representative surface soil samples to estimate mean concentrations of residues of high explosives at two types of potential source zones: (1) an area near a low-order [partial] detonation of an 81-mm mortar and (2) an artillery/mortar target. Soil sampling near the low-order detonation revealed the presence of potential "hot spots" and showed that the concentrations of RDX and TNT ranged over five orders of magnitude. The range of concentrations was reduced to a factor of about 60 when randomly collected $25-$ increment composite samples were collected within this area. The range reduced further to about a factor of three for four simulated (i.e., existing discrete values) 25 -increment systematically derived composite samples. Thus a vast improvement in the repeatability of replicate samples can be achieved using composite sampling approaches. Composite samples collected around a target showed that the distribution of energetic residues was random and overall the concentrations were much lower than around the partially detonated round.

\begin{tabular}{|c|c|c|c|c|c|}
\hline 15. SUBJECT TERMS & \multicolumn{2}{|c|}{$\begin{array}{l}\text { Energetic residues } \\
\text { Impact range }\end{array}$} & Source zones & & \\
\hline \multicolumn{3}{|c|}{ 16. SECURITY CLASSIFICATION OF: } & 17. LIMITATION OF & 18. NUMBER & 19a. NAME OF RESPONSIBLE PERSON \\
\hline a. REPORT & b. ABSTRACT & c. THIS PAGE & & & 19b. TELEPHONE NUMBER (include area code) \\
\hline $\mathrm{U}$ & $\mathrm{U}$ & $\mathrm{U}$ & $\mathrm{U}$ & 45 & \\
\hline
\end{tabular}

